\title{
Das Spießerverdikt. Invektive Umordnungen des Sozialen seit der Romantik
}

\author{
Sonja Engel · Dominik Schrage
}

Angenommen: 29. Juli 2021 / Online publiziert: 24. August 2021

(C) Der/die Autor(en) 2021

Zusammenfassung „Spießern“ wird im Deutschen in herabsetzender Absicht Engstirnigkeit und eine konformistische Lebensweise unterstellt. Der Aufsatz fasst das Spießerverdikt als ein kommunikatives Muster, das in Auseinandersetzungen um die Deutung der sozialen Ordnung eingesetzt wird, indem es die Angehörigen der Mitte als Repräsentanten der bestehenden Ordnung attackiert. Varianten des Spießerverdikts zirkulieren im deutschen Sprachraum seit dem frühen 19. Jahrhundert. Sie entwerfen gegenhegemoniale Sinnhorizonte der gesellschaftlichen Verortung und Legitimation. Um ihre Funktion bei der Erzeugung und In-GeltungSetzung von Vorstellungen sozialer Ordnung sichtbar zu machen, wird die Herausbildung des kommunikativen Musters anhand dreier Varianten im deutschsprachigen Raum nachgezeichnet: der Philistersatire in der Romantik, der Kleinbürgerkritik in der durch Marx und Engels geprägten Klassentheorie und der antibürgerlichen Selbstinszenierung der Bohemiens. Damit wird eine kultursoziologische Perspektive auf sozialen Wandel eingenommen, die die diskursive Praxis der Herabsetzung als Faktor von Konflikten in Zeiten gesellschaftlicher Umbrüche fokussiert.

Schlüsselwörter Kultursoziologie $\cdot$ Spießer · Mitte der Gesellschaft · Sozialer Wandel · Historische Diskursanalyse

\footnotetext{
Sonja Engel $(\triangle)$

SFB 1285 - Invektivität, Technische Universität, 01062 Dresden, Deutschland

E-Mail: sonja.engel@tu-dresden.de

Dominik Schrage

Institut für Soziologie, Lehrstuhl für Soziologische Theorien und Kultursoziologie, Technische

Universität, 01062 Dresden, Deutschland

E-Mail: dominik.schrage@tu-dresden.de
} 


\title{
The anti-philistine verdict. Invectives to rearrange the social order since the romantic era
}

\begin{abstract}
In German, "Spiesser" (philistine) is used as a pejorative term designating a narrow-minded and conformist way of life. In this paper, the term "Spießerverdikt" (philistine verdict), whereby the members of the middle class are attacked as representatives of the existing order, is conceptualised as a communicative pattern used in disputes about the interpretation of the social order. Various forms of the "Spiesserverdikt" have been circulating in the German-speaking area since the early 19 th century, creating counter-hegemonic horizons of meaning for social positioning and legitimation. In order to assess their function in the creation and validation of ideas of social order, the paper traces the development of the "Spiesserverdikt" on the basis of three variants: the philistine satire in the Romantic era, the criticism of the petty bourgeois in the class theory shaped by Marx and Engels and the bohemian anti-bourgeois self-portrayal. Working from a cultural-sociological perspective on social change, it is shown how the discursive practice of disparagement is used in conflicts about the interpretation of the (existing and desired) social order and can become a factor of societal upheaval itself.
\end{abstract}

Keywords Cultural Sociology $\cdot$ Philistines $\cdot$ Middle class $\cdot$ Social change · Historical discourse analysis

\section{Le dénigrement du petit-bourgeois. L'invective comme vecteur de transformation sociale depuis l'époque romantique}

Résumé En allemand, «Spießer » (petit-bourgeois) désigne de manière péjorative des personnes à qui on attribue un mode de vie borné et conformiste. Dans cet article, le dénigrement du petit-bourgeois est conceptualisé comme une figure communicationnelle utilisée dans les luttes pour l'interprétation de l'ordre social consistant à attaquer les membres de la classe moyenne comme représentants de l'ordre établi. Des variantes de ce dénigrement du petit-bourgeois circulent dans l'espace germanophone depuis le début du XIXe siècle. Elles esquissent des horizons de sens contre-hégémoniques pour le positionnement et la légitimation sociale. Pour mettre en évidence la fonction de cette figure communicationnelle dans la production et la consécration de conceptions de l'ordre social, sa formation est retracée à partir de trois variantes identifiables dans l'espace germanophone: la satire des philistins à l'époque romantique, la critique du petit bourgeois dans la théorie des classes inspirée de Marx et Engels et la mise en scène de la bohème comme antibourgeoisie. Cette mise en perspective du changement social par la sociologie de la culture se focalise sur la pratique discursive de la dépréciation comme facteur de conflit dans les périodes de bouleversements sociaux.

Mots-clés Sociologie de la culture · Petit-bourgeois · Classe moyenne · Changement social $\cdot$ Analyse de discours historiques 


\section{Einleitung}

Die im Deutschen geläufige beleidigende Bezeichnung Spieß(bürg)er erfuhr ihre letzte Hochkonjunktur im Kontext der antikonformistischen Bewegungen in den 1960er- und 1970er-Jahren. Hier brachte sie einen sozialen und politischen Konflikt zum Ausdruck, in dem sich das Selbstverständnis der Alternativmilieus als progressive Gegenkultur zu der ,spießbürgerlichen“ Majoritätsgesellschaft herausbildete. Die Spießerbeschimpfung ist aber auch die Blaupause aktuellerer Variationen wie jener des „Wutbürgers“ (Kurbjuweit 2010), des „Bionade-Biedermeiers“ (Sußebach 2007) und des „Alternativspießers“ (Misik 2006) sowie in neuester Zeit des „Corona-Spießers“ (Feldenkirchen 2020), mit denen bestimmte Lebensweisen in herabsetzender Weise charakterisiert werden. Bis heute erweisen sich Spießerbeschimpfungen also als höchst alltagstauglich. Allerdings hat dieses Phänomen in der Soziologie bisher wenig Aufmerksamkeit erfahren, wie sich an der spärlichen Literatur zum Thema zeigt (außer Kajetzke 2010). Unseres Erachtens zu Unrecht, denn die Betrachtung dieser kommunikativen Akte schärft den Blick für eine wirkungsreiche kulturelle Logik der Moderne. Diese zeichnet sich dadurch aus, dass das Bestehende und Etablierte delegitimiert und das Neue und Innovative als erstrebenswert markiert wird. Dementsprechend können sich Nonkonformist*innen als Avantgarde und Trägergruppe des Fortschritts inszenieren, während „Spießer*innen“ als Verwalter*innen und Bewahrer*innen eines bereits veralteten Normensystems mit Stagnation oder gar Regression assoziiert und abgewertet werden. Die Spießerbeschimpfungen erweisen sich mithin als zentrales Untersuchungsobjekt in der Genealogie des Nonkonformismus, die es noch historisch-soziologisch zu erschließen gilt.

Wie wir in diesem Beitrag zeigen, liegt den ephemeren Akten der Spießerbeschimpfung im Alltag ein komplexes und historisch tradiertes kommunikatives Muster zugrunde, dessen historische Genese im 19. Jahrhundert situiert ist und das wir das Spießerverdikt nennen. In diesem Sinne verwenden wir den Begriff im Singular, während wir dem Formenwandel dieses Musters mit der Bezeichnung Varianten Rechnung tragen. Solche Varianten stellen, so unsere Ausgangsbeobachtung, im 19. Jahrhundert die Bezeichnungen Philister und Kleinbürger dar, die neben der Bezeichnung Spießbürger ${ }^{1}$ das Bedeutungsspektrum der heute geläufigen Spießerverdikte wesentlich mitprägten (vgl. Stein 1985). Ihnen allen ist gemeinsam, dass sie ein Desidentifikationsangebot liefern: Sie charakterisieren eine verbreitete Lebensweise und rufen zugleich dazu auf, diese zu verachten. Weil sie in den öffentlichen Diskurs eingespeist werden und dort zirkulieren, gehen sie in den kollektiven Wissensbestand ein und können in Auseinandersetzungen um die „richtige Lebensweise“ eingesetzt und aktualisiert werden. Im Fokus stehen dabei jeweils die Lebensweisen und Haltungen der herabgesetzten Gruppen; ihnen werden Eigenschaften wie

\footnotetext{
1 Dass die Bezeichnungen alle nur im Maskulinum verwendet werden, ist dabei nicht nur auf die lange währende Verwendung des generischen Maskulinums zurückzuführen. Den vergeschlechtlichten Konstituenten der Figur des Spießers nachzugehen, ist jedoch ein eigenes Thema, das hier nicht weiter verfolgt werden soll. Da „Philister“, „Klein-“ und „Spießbürger“ in diesem Beitrag als Bezeichnungen verwendet werden, die wir dem Diskurs entnehmen und wir damit keine realen Personen(gruppen) benennen, übernehmen wir unverändert die männliche Form.
} 
Passivität, Konformität, Neophobie und Konservatismus zugeschrieben, womit entsprechend der oben erläuterten Logik ihre Abwertung betrieben wird.

Seine soziologische Relevanz, so argumentieren wir, wird deutlich, wenn das Spießerverdikt als eine Praktik der Herabsetzung verstanden wird, die als Kampfmittel in Auseinandersetzungen um die Bewertung spezifischer Positionen in der sozialen Ordnung fungiert. Das Spießerverdikt erweist sich auch in dieser Hinsicht als eine genuin moderne Invektive, da es nur unter spezifischen historischen Bedingungen Wirkmacht entfalten kann. Es entsteht in einem historischen Kontext, in dem durch die Ideen der Aufklärung und soziale Umbrüche die bestehende Ordnung an Überzeugungskraft verliert und Alternativen denk- und sagbar werden. Auch im Zusammenhang eines durch Neuerungen geprägten Wandels der Medien entsteht ein öffentlicher und bürgerlich geprägter Diskursraum, in dem Auseinandersetzungen über gesellschaftliche Verhältnisse sowie erwünschte und unerwünschte Entwicklungen stattfinden und dabei Vorstellungen sozialer Ordnung artikuliert werden. Während Theorien der Modernisierung häufig darauf beruhen, den sozialen Wandel primär als einen Strukturwandel zu beschreiben, ist unser Anliegen, die diskursiven Deutungskonflikte zu betrachten, die diesen Wandel prägen.

Die Varianten des Spießerverdikts im 19. Jahrhunderts sind in solche Deutungskonflikte insofern involviert, dass sie Konflikte um die Bewertung der gesellschaftlichen „Mitte“ und ihrer Repräsentanten sowie um ihre Bedeutung für die zukünftige Entwicklung der Gesellschaft zum Ausdruck bringen oder auch evozieren. Die hier auf Grundlage dieser Annahmen entwickelte soziologische Perspektive auf das Spießerverdikt zielt somit vor allem darauf, dieses als eine Praktik der Herabsetzung von Angehörigen der sozialen Mitte historisch nachzuvollziehen: Spießerverdikte thematisieren spezifische Formen der bürgerlichen Lebensweise und verknüpfen die Eigenschaften des Konformismus und Konservatismus mit Positionen in der Mitte der Gesellschaft. Damit unterwerfen sie in bemerkenswerter Weise dasjenige soziale Stratum einer Schmähung, das sich seit dem 18. Jahrhundert im Aufstieg befindet und für viele als Hoffnungsträger für eine egalitärere Ordnung fungiert. So stellten Angehörige der sozialen Mittellagen aus liberaler Perspektive die von Adel und Unterschicht abgegrenzten Impulsgeber gesellschaftlichen Fortschritts dar - schon Hume sprach vom ,middling rank of men, who are the best and firmest basis of public liberty“ (Hume 2003 [1752], S. 112). Bis heute gibt es eine diskursiv wirkmächtige Deutung der Mitte als Zentrum der Gesellschaft und als Ort des Ausgleichs (vgl. u.a. Marg 2014).

Die abwertende Qualifizierung der Mittellagen, die in den Diskurs eingespeist wird, ist dabei immer auch eingebunden in Auseinandersetzungen um die Deutung der sozialen Ordnung als Ganze, da in den Spießerverdikten andere soziale Positionen - zum Beispiel jene, die in einem metaphorischen ,Unten“ oder „Außen“ verortet sind - ebenfalls mit jeweils spezifischen Bewertungen belegt werden. Da Angehörige der gesellschaftlichen Mitte als Repräsentanten der bestehenden Ordnung mit stereotypen pejorativen Merkmalen versehen werden, so unsere Beobachtung, werden im Gegenzug solche Lebensweisen und Identitäten hoch bewertet, die in der imaginierten Ordnung bislang sozialräumlich peripher positioniert sind. Indem mit Hilfe der Verdikte die gesellschaftliche Mitte als Ort der Stagnation, der überlebten Konvention und der konformistischen Langeweile markiert und zur 
Desidentifikation mit den damit assoziierten Milieus und Lebensweisen aufgerufen wird, erscheinen die Lebens- und Handlungsformen der Angreifenden zugleich als ethisch superior und attraktiv. Zieht sich diese räumliche Positionierungspraxis durch sämtliche der im Folgenden untersuchten historischen Varianten des Spießerverdikts, so offenbaren sich, wie wir zeigen werden, mit Blick auf die zeitlichhistorische Dimension aber durchaus auch markante Unterschiede. ${ }^{2}$

Das Interesse unseres Beitrages richtet sich mithin auf die Frage, wie die historischen Varianten des Spießerverdikts in Deutungskonflikten über die Bewertung der sozialen Positionen in der Mitte und der Nicht-Mitte die unterstellte Funktion erfüllen, wie sie jeweils eigene Kriterien der gesellschaftlichen Verortung und Legitimation artikulieren sowie Lebensweisen und ihnen zugeordnete soziale Positionen be- und umbewerten. Zunächst stellen wir die Perspektive unserer Untersuchung vor, die sich erstens aus kultursoziologischen Ansätzen speist, die historischen Wandel als einen Effekt von Deutungskämpfen verstehen, und zweitens aus dem Konzept der Invektivität (Abschnitt 2). Im Hauptteil des Beitrages (Abschnitt 3) zeichnen wir die Herausbildung des kommunikativen Musters im 19. Jahrhundert anhand dreier Varianten im deutschsprachigen Raum nach, in deren Zentrum jeweils eine invektierte Sozialfigur der Mitte steht: die Philistersatire im Umkreis der Romantik, die Kleinbürgerkritik in der durch Marx und Engels geprägten Klassentheorie sowie die antibürgerliche Selbstinszenierung der Boheme. Abschließend zeigen wir in einer die Varianten des Spießerverdikts vergleichenden Zusammenfassung, inwiefern sie mit ihren jeweils unterschiedlichen Abwertungen von Repräsentanten der sozialen Mitte ein Reservoir von Herabsetzungspraktiken erzeugt haben, die bis heute in den Spießerbeschimpfungen selektiv reproduziert und reaktualisiert werden.

\section{Theoretische Perspektivierungen, Vorgehensweise und zentrale Begriffe}

\subsection{Das Spießerverdikt als Kampfmittel in Konflikten um die Deutung der sozialen Ordnung}

Während Spießerbeschimpfungen, wie eingangs erwähnt, in der Soziologie bisher nicht behandelt worden sind, wird auch der Figur des Spieß(bürg)ers selbst kaum Aufmerksamkeit der Disziplin zuteil. Die Figur des Philisters ist in den Literaturwissenschaften untersucht worden (vgl. Bunia et al. 2012), zum Spießer gibt es eine begriffsgeschichtlich orientierte Abhandlung (Hahn 2009). Generell scheinen diese Bezeichnungen jedoch kaum dazu geeignet, Bevölkerungsgruppen auf sozialwissenschaftliche Weise zu bestimmen. Dagegen ist der Kleinbürger bzw. das Kleinbürgertum noch häufiger in sozialwissenschaftlicher Literatur zu finden. So dient

\footnotetext{
2 Es soll damit nicht behauptet werden, dass das hier beschriebene kommunikative Muster, das die Aufwertung der jeweils eigenen Lebensweise mit der Abwertung anderer Lebensweisen verknüpft, erst im 19. Jahrhundert zum Einsatz kommt. Auch Satiren auf Konformisten und die Majoritätsgesellschaft haben historische Vorläufer, siehe beispielsweise François Villon. Die Spießerverdikte des 19. und 20. Jahrhunderts zeichnen sich jedoch dadurch aus, dass die eigene Lebensweise als superior markiert wird, weil sie dem Fortschritt dient. Dies wird erst vor dem Hintergrund einer modernen Forschrittssemantik plausibel.
} 
er einigen durchaus im Anschluss an die Klassentheorie des 19. Jahrhunderts als tauglicher Begriff im Rahmen von Sozialstrukturanalysen (vgl. Eder 1989), von anderen wird er eher sozialpsychologisch als Bezeichnung für einen Mentalitätstypus sowie ein damit verbundenes, spezifisches Set von kulturellen Praktiken verwendet (vgl. Schilling 2003). Diese Beiträge sind dabei selbstverständlich selbst in den hier interessierenden Diskurs und die Konflikte um die Deutung der gesellschaftlichen Mitte involviert. Seinen Charakter als ,politische[r] Kampfbegriff“ (Franke 1988, S. 12) kann der Begriff des Kleinbürgers deshalb auch in den wissenschaftlichen Betrachtungen nicht vollständig abstreifen.

Die hier vorgeschlagene Perspektive auf das Spießerverdikt ist dementsprechend nicht darauf ausgerichtet zu bestimmen, was Spießer, Philister oder Kleinbürger ,eigentlich“ sind. Vielmehr versuchen wir aus historisch-wissenssoziologischer Sicht zu verstehen, inwiefern die Herabsetzung von Angehörigen sozialer Mittellagen durch das Spießerverdikt an der Etablierung und Veränderung von Ordnungsvorstellungen beteiligt war und ist. ${ }^{3}$ Dabei verorten wir uns innerhalb des kultursoziologischen Paradigmas, das gesellschaftlichen Wandel in der Moderne als einen Prozess versteht, der durch Brüche und Ambivalenzen gekennzeichnet ist und das Augenmerk auf ,die konflikthafte und in diesem Sinne unebene und offene, nicht-lineare Transformation von Praktiken und Diskursen“ legt (Bonacker und Reckwitz 2007, S. 13).

Anknüpfend an Foucault und andere poststrukturalistische Denker*innen begreifen wir sozialen Wandel als in Wechselwirkung mit diskursivem Wandel stehend. In einer solchen Perspektive wird Wandel nicht als eine „der Geschichte oder den Strukturen selbst inhärente Logik“ aufgefasst, sondern als ,fundamental von gesellschaftlichen Kräfteverhältnissen [...] sowie sozialen Kämpfen und Auseinandersetzungen abhängig“ verstanden (Gertenbach 2008, S. 220), sodass Deutungskonflikte eine hohe Aufmerksamkeit erfahren. Diese Perspektive erweitern wir um das Konzept der „Invektivität“ und beanspruchen damit auch, soziologische Konflikttheorien (vgl. Bonacker 2008, S. 207 ff.) kultursoziologisch zu erweitern.

Invektivität ${ }^{4}$ bezeichnet jene Aspekte von Kommunikation, die das Potenzial in sich bergen, ein reales oder imaginäres Gegenüber herabzusetzen, auszugrenzen und zu beleidigen. Sein Gebrauch eröffnet den Zugriff auf ein ,gesellschaftliches Fundamentalphänomen, das Affekte, strategische Kalküle und symbolische Geltungsansprüche in spezifischer Weise verknüpft“" (Ellerbrock et al. 2017, S. 5). Der damit verbundene empirische Fokus richtet sich in einer Perspektive des epochen- und kulturübergreifenden Vergleichs auf kommunikative Akte der Herabsetzung, die in

\footnotetext{
3 Die Invektiven gegen Angehörige sozialer Mittellagen sind ebenfalls eng mit der diskursiven Konstitution von Bürgerlichkeit verflochten, da sie auch auf das Selbstverständnis des Bürgertums als gesellschaftstragende Schicht reagieren (vgl. zum Bürgertum im 19. Jahrhundert u.a. Kocka 2001). In sozialhistorischen Forschungen zum 19. Jahrhundert werden auch die bürgerlichen Lebensweisen in der Regel ausführlich thematisiert (vgl. u.a. Schulz 2014), Kritiken an Bürgerlichkeit erfahren allerdings weniger Aufmerksamkeit (vgl. aber Siegrist 1999).

${ }^{4}$ Der Begriff der Invektivität ist ein Neologismus, der im Rahmen des Sonderforschungsbereichs 1285 „Invektivität. Konstellationen und Dynamiken der Herabsetzungen“ an der Technischen Universität Dresden interdisziplinär fruchtbar gemacht worden ist und in dessen Rahmen wir unsere Forschungen durchführten. Die Darstellung der Varianten des Spießerverdikts in Abschnitt 3 ist eine Art „Blütenlese“ der Analyseergebnisse unseres Teilprojekts. Für eine ausführlichere Darstellung unserer Forschungsergebnisse vgl. Engel und Schrage 2021.
} 
sozialen Situationen beobachtbar sind oder sich in mediatisierten Kommunikationen und diskursiven Thematisierungen manifestieren. Diese beobachtbaren kommunikativen Akte werden, den lateinischen Begriff der Schmährede verallgemeinernd, als Invektiven bezeichnet. In diesem Sinne sind auch die in diesem Beitrag betrachteten Spießerverdikte als Invektiven zu verstehen. Beim Spießerverdikt, so wird dabei im Folgenden argumentiert, handelt es sich um eine Art der Invektive, deren Effekte auf die soziale Ordnung wie schon eingangs gesagt vor allem darin liegen, dass mit ihr die Mitte der Gesellschaft problematisiert wird. In den Fokus gerät damit insbesondere ihre Bedeutung als normgebende Instanz. Während im hegemonialen Diskurs diese soziale Funktion mehr oder minder explizit als legitim erachtet wird, machen die Invektiven sie zum Gegenstand des Konflikts. Sie zielen auf Dynamisierungen und Veränderungen des gesellschaftlichen Normengefüges und artikulieren diese im Diskurs im Modus des Invektiven.

Das Konzept der Invektivität macht es möglich, die für das Spießerverdikt typische Form der Herabsetzung auf eine Weise zu fassen, die spezifischer ist als geläufige Konzepte wie Diskriminierung, sprachliche Gewalt oder symbolische Verletzung (Schrage 2021). Diese bieten für die Praktik der Spießerschmähung deshalb keinen geeigneten Zugriff, weil der Spießer keine soziale Kategorie ist, der ernsthaft eine strukturelle Benachteiligung zugesprochen werden könnte. Auch als sprachliche Gewaltakte könnten Spießerverdikte nur in einem allzu weiten Verständnis verstanden werden. Zwar mag die Beschimpfung als Spießer in bestimmten Interaktionssituationen als verletzend wahrgenommen werden und die Charakterisierung als Spießer der Legitimation einer sozialen Exklusion dienen. Jedoch entfalten unserer Ansicht nach insbesondere die in Textform überlieferten und zum Teil kanonisierten Spießerverdikte, die das Material dieses Beitrags liefern, ihre Effektivität auf einer anderen Ebene, nämlich als diskursive Strategie in Deutungskonflikten um die Ordnung des Sozialen.

Für unsere Frage nach der Funktionsweise des Spießerverdikts erweist sich diese Perspektivierung insbesondere deshalb als fruchtbar, weil sie die soziologische Sicht auf die Kategorie der gesellschaftlichen Mitte erweitert. Diese verstehen wir nicht als kriteriengestützt-positive sozialwissenschaftliche Analysekategorie, sondern als öffentliches Deutungsmuster, das nicht nur von einer Vielzahl von Akteuren mit unterschiedlichsten Wertungen und Signifikaten gebraucht wurde und wird, sondern sich auch historisch gewandelt hat. Mit dem Fokus auf das Spießerverdikt zeigt sich sozialer Wandel damit nicht nur als ein sozialstruktureller Prozess der Umschichtung, sondern auch als eine Kette aufeinanderfolgender Deutungskonflikte, in denen um die Einordnung und Bewertung der sozialen Mitte gestritten wird (vgl. Fischer 2007). Kurzum: Was „Mitte“ heißt, ist gesellschaftlich umstritten, und der Streit darum hat eine Geschichte. In diesem Rahmen erhält das Spießerverdikt die Funktion eines Kampfmittels, das alternative Vorstellungen von Mitte und Peripherie und damit von sozialer Ordnung in Geltung setzt.

\subsection{Positionierungspraxis und Ordnungsvorstellungen}

Unsere kultursoziologische Perspektive haben wir in unserem Forschungsprojekt in einer an Verfahren der sozialwissenschaftlichen, insbesondere der wissenssoziologi- 
schen Diskursanalyse orientierten Vorgehensweise umgesetzt (Keller 2011; Keller et al. 2001; Bosančić und Keller 2016; Bührmann und Schneider 2013). Diese wurden für die besonderen Herausforderungen der Analyse eines historischen Gegenstandsbereiches angepasst; zudem wurden Anregungen aus der historischen Diskursanalyse aufgenommen (Landwehr 2008; Füssel und Neu 2010; Klöppel 2010). Ausgehend von vorhandener Literatur zum Thema und eigenen Recherchen haben wir eine Fülle von Texten aus literarischen, politischen und gesellschaftstheoretischen sowie ästhetischen Publikationen aus dem 19. Jahrhundert zum Korpus unserer Analyse zusammengestellt. Für diesen Beitrag ist Diskursmaterial ausgewählt worden, das sich bei der Bearbeitung unserer Fragestellung als besonders relevant erwiesen hat, da in den hier versammelten Passagen verbreitete Formen des Spießerverdikts besonders verdichtet erkennbar werden und sich damit dessen Funktionsweise konturiert. Am ausgewählten Material lässt sich exemplarisch zeigen, wie die Spießerverdikte als Desidentifikationsangebote diskursiv konstituiert und als Kampfmittel in Konflikten um die Durchsetzung von Deutungen der sozialen Ordnung eingesetzt werden.

Dass wir das Spießerverdikt als ein Desidentifikationsangebot beschreiben, beruht auf einer wissenssoziologischen Überlegung, derzufolge es seinen herabsetzenden Sinn ausgehend von einer „Ideologie“ erhält, verstanden im Sinne Karl Mannheims als Verabsolutierung der partikularen Weltsicht einer sozialen Gruppe (Mannheim 1982). Die Besonderheit des Spießerverdikts liegt nun darin, dass es Lebensweisen und Wertvorstellungen von in der Mitte situierten Gruppen öffentlich als minderwertig qualifiziert und damit jene, die sie an den Tag legen, abwertet. Allgemein gesagt handelt es sich um Lebensweisen, die weit verbreitet sind, hegemonialen Konventionen entsprechen und geltende Normen nicht infrage stellen. Konformität wird in den Spießerverdikten durchgängig mit Mediokrität, Passivität und Konservatismus gleichgesetzt und vor dem Hintergrund eines alternativen, als dissident verstandenen Normenhorizontes bewertet, der typischerweise Nonkonformität und Progressivität nobilitiert. Insofern werden im Spießerverdikt auch implizit oder explizit Entwürfe alternativer Lebensweisen und Normenhorizonte mitgeführt, die als superior bewertet werden: Wenn Philister, Kleinbürger und Spießer als passiv, konform, neophob und konservativ dargestellt werden, korrespondiert dies mit der Selbstbeschreibung der Schmähenden als aktiv, nonkonform, welt- und zukunftsoffen sowie zukunftsfähig, also als progressiv oder gar revolutionär. Die Defizienz der Lebensweisen von Philistern, Kleinbürgern und Spießern zu konstatieren, bedeutet deshalb nicht nur sie abzuwerten und herabzusetzen, sondern auch einerseits die Normen zu kritisieren, an denen sie sich vermeintlich orientieren, sowie andererseits entgegengesetzte Normen zu propagieren. Die charakteristische Verbindung dieser beiden Operationen führt uns dazu, das kommunikative Muster des Spießerverdikts als ein Desidentifikationsangebot zu bezeichnen: Es suggeriert zum einen, dass man so, wie die Spießer sind, nicht sein sollte, und stellt zum anderen eine plausible Alternative für die Orientierung der eigenen Lebensweise zur Verfügung.

Die in der Gesellschaft vertretenen Lebensweisen werden auf diese Weise charakterisiert und durch Bewertungsakte hierarchisiert, woraus auch eine soziale Hierarchisierung ihrer Trägergruppen resultiert. Den Repräsentanten des Konformen und Konservativen wird im Spießerverdikt somit eine niedere Position in dem so etablierten Gefüge von Positionen zugewiesen: Sie werden herabgesetzt. Dies kann in 
Form einer Umwertung bestehender Positionsgefüge geschehen, aber auch dadurch, dass neue Positionen ins Spiel gebracht werden. Das Spießerverdikt verstehen wir deshalb als eine Praktik der sozialen Positionierung, mithin als eine Verortung in einem Modell sozialer Ordnung, das selbst durch diese Akte der Positionierung (mit)erzeugt wird. Im Folgenden bezeichnen wir diese in den Diskurs eingebrachten Ordnungsmodelle kurz als Ordnungsvorstellungen, um ihren diskursiven Charakter hervorzuheben und zu verdeutlichen, dass es sich nicht um Aussagen über eine faktisch existierende soziale Ordnung handelt. Entsprechend fassen wir sowohl diese Ordnungsvorstellungen als auch die auf sie bezogenen Akte sozialer Positionierung als Bestandteile des untersuchten Diskursgeschehens auf.

Indem wir den Fokus auf diskursive Positionierungspraktiken legen, nehmen wir eine Perspektive ein, die sich von dem in der Diskursforschung weitaus verbreiteteren Fokus auf Prozesse der „Subjektivierung“ bzw. der „Subjektivation“ unterscheidet (vgl. etwa Bröckling 2007; Reckwitz 2006; Gelhard et al. 2013). Das ist vor allem in der Beschaffenheit unseres Gegenstands als Desidentifikationsangebot begründet. Während mit dem Konzept der Subjektivierung auf die Rekonstruktion der ,soziohistorische[n] Form typischer Akteursperspektiven“ (Schrage 2012, S. 82) sowie der Prozesse ihrer Aneignung und ihrer Machtwirkungen fokussiert wird, stellen wir dem Leitkonzept des Forschungsverbundes folgend invektive Kommunikationsakte in den Mittelpunkt. Als desidentifikatorisches Diskursereignis interessiert uns das Spießerverdikt zunächst in seiner Performativität, und diese besteht v.a. in der Zuweisung niedrig bewerteter sozialer Positionen in den diskursiv entworfenen Ordnungsmodellen. Die sozialen Funktionen und Effekte der Spießerverdikte lassen sich mit dem Fokus auf diskursive Positionierungspraktiken unserer Ansicht nach am deutlichsten aufzeigen. Zwar behandeln beide Perspektiven die von ihnen untersuchten diskursiven Praktiken - seien sie als Subjektivierung oder als Positionierung gefasst - im Anschluss an Foucault als „Praktiken [...], die systematisch die Gegenstände bilden, von denen sie sprechen“ (Foucault 1981, S. 74). Im Falle der Spießerverdikte ist der besonders interessante Gegenstand, der erzeugt wird, jedoch nicht eine Subjektform, sondern ein durch Invektiven in Geltung gesetztes Gefüge sozialer Positionen, d.h. soziale und geschichtliche Ordnungsvorstellungen. Das schließt die Frage nach ihren subjektivierenden Wirkungen nicht aus, denn die Spießerverdikte vermitteln durchaus Normen der richtigen und der falschen oder lächerlichen Lebensführung und können somit normative Kräfte auf Individuen ausüben. So rufen Spießerverdikte auch zu einer Identifikation mit den Invektierenden auf, die jedoch unter Umständen durch eine spielerische und ephemere Übernahme der nahegelegten Perspektive der Nonkonformen befriedigt werden kann. ${ }^{5}$ Dies steht jedoch nicht im Zentrum unseres Beitrags.

Die für das Spießerverdikt charakteristischen, ineinandergreifenen Fremd- und Selbstpositionierungen können auch in Anlehung an Pierre Bourdieu als soziale Distinktionsakte beschrieben werden, deren Besonderheit allerdings darin liegt, dass in ihnen die sozialen Positionen der Akteure nicht allein in latenter Form - als im Habitus sedimentierte Dispositionen und Erfahrungsmuster - zum Ausdruck kommen.

\footnotetext{
${ }^{5}$ Insofern können die Figur des Spießers und ihre Angreifer auch als Rollenangebote verstanden werden, die temporär und situativ leicht übernommen, ebenso leicht aber auch wieder abgelegt werden können.
} 
Vielmehr haben wir es mit Praktiken der „Klassifizierung“ zu tun, die sich explizit gemachter Vorstellungen der sozialen Ordnung bedienen, wenn sie anderen einen Ort und einen Wert in dieser Ordnung zuschreiben und diese selbst in Anspruch nehmen. Anders als im analytischen Modell des sozialen Raumes, das Bourdieus Theorie von der Reproduktion der sozialen Ordnung zugrunde liegt und das als wissenschaftliches Modell ,auf dem Papier“" scharf von den Alltagstheorien der Akteure unterschieden wird (Bourdieu 1982, 1998, S. 13 ff.), werden die Vorstellungen der sozialen Ordnung beim Spießerverdikt in den Deutungen der Akteure explizit. Eben dies ermöglicht den Einsatz der Spießerverdikte als besondere „Mittel“, „Waffen“ und „Einsätze im Kampf um die Klassifizierungen“ (Bourdieu 1998, S. 117).

Am Spießerverdikt wird somit sichtbar, dass die Abwertung etablierter Vertreter der sozialen Mitte oft mit der Inanspruchnahme eines privilegierten symbolischen Orts innerhalb einer im Diskurs konstruierten sozialen Ordnung einhergeht. Wir haben es demnach mit Konflikten zu tun, in denen um die in der Gesellschaft gültige Deutung der sozialen Ordnung gekämpft wird. Sie wird dann jeweils für die Bemessung von Prestige herangezogen, und in die Konflikte werden einander widerstreitende Maßstäbe von Prestige eingebracht. Um diesen Aspekt der im Spießerverdikt sichtbar werdenden Klassifizierungspraktiken zu berücksichtigen, müssen also die im Diskurs entfalteten Ordnungsvorstellungen vom analytischen Modell des sozialen Raumes im Sinne Bourdieus unterschieden und eigens rekonstruiert werden. Denn die umstrittenen Ordnungsvorstellungen - verstanden als Rahmen, auf den sich die Positionierungsakte beziehen, sowie als Kriterienkatalog für die Hochund Niedrigbewertung sozialer Positionen - lassen sich erst aus der Rekonstruktion des im Diskurs in Geltung gesetzten normativen Registers herausarbeiten.

Das Spießerverdikt kann vor diesem Hintergrund somit als Moment eines komplexen, konfliktreichen Diskursgeschehens verstanden werden, das die gesellschaftlich geltenden Auffassungen vom ,richtigen Leben“ und von sozialer Ordnung betrifft. Ein Anliegen des Beitrags ist es demnach, aus den im Laufe des 19. Jahrhunderts nacheinander entstehenden Spießerverdikten die für sie maßgeblichen Ordnungsvorstellungen zu rekonstruieren, wobei ein besonderes Augenmerk auf die Kriterien der Bewertung und die Maßstäbe der Prestigebemessung gerichtet wird. Dabei werden zugleich Veränderungen und Kontinuitäten zwischen den kommunikativen Mustern der drei Varianten des Spießerverdikts herausgestellt, um Anschlüsse und Brüche herauszuarbeiten.

\section{Die Spießerverdikte des 19. Jahrhunderts}

\subsection{Die Philistersatire der Romantik}

Mit den Philistersatiren der Romantik, einem Produkt des literarischen Feldes, entsteht an der Wende zum 19. Jahrhundert eine erste Variante des Spießerverdikts. Die Philisterfigur „kursierte“, so Stein, ,als ein gängiges Klischee in der romantischen Literatur" (Stein 1985, S. 10). Dementsprechend haben sich bisher vorrangig Literaturwissenschaftler*innen mit dieser Figur und der zugehörigen Semantik beschäftigt. Die Herausgeber eines ertragreichen Sammelbandes zur Philisterfigur 
aus literatur- und kulturwissenschaftlicher Sicht konstatieren jedoch auch in diesen Disziplinen eine Diskrepanz zwischen der weiten Verbreitung der Sozialfigur des Philisters in der zeitgenössischen Literatur einerseits und der geringen Aufmerksamkeit der Forschung andererseits (Bunia et al. 2012, S. 13). In ihrem Band nehmen sie u.a. eine grobe Systematisierung des semantischen Feldes und auch der sozialen Funktionen dieser Bezeichnung vor, die sich im Laufe des 19. Jahrhunderts verändert und anreichert. Dazu benennen sie fünf verschiedene Problemkomplexe, mit denen die Philistersemantik verbunden ist: erstens die „Unterscheidung Eigenes/Anderes“, zweitens die Thematisierungen von ,soziale[r] Mobilität“, drittens von „Gewalt“ und viertens von „Geschlecht“ sowie fünftens von „Bildung“ (ebd., S. 16 ff.). Damit werden zentrale Dimensionen sozialer Ordnung benannt. Zwar enthält die Verwendung des Philisterbegriffs den Autor*innen zufolge ,regelmäßig einen herabwürdigen Aspekt“ (ebd., S. 18), jedoch verfolgen sie diesen nicht weiter hinsichtlich der hier interessierenden Frage, welche Ordnungsvorstellungen diese Praktik der Herabsetzung impliziert und verbreitet.

Für diese Frage ist von besonderem Interesse, dass die Philistersatire durch $\mathrm{Zu}$ schreibungen der Mediokrität, des Konformismus und des Konservatismus eine Position konturiert, die den Philister in der sozialen Mitte verortet. Dies ist allerdings noch nicht mit der Vorstellung einer modernen Sozialstruktur verbunden, sondern speist sich vielmehr aus der Verachtung alles Gewöhnlichen und Mittelmäßigen. ${ }^{6}$ In der Romantik werden in der Philisterfigur zwei bereits verbreitete Bedeutungen der Bezeichnung „Philister“ kombiniert. Im literarischen Feld repräsentiert sie zunächst die dilettantische Gegenfigur zu den kunstschaffenden Genies und „Musensöhnen“. Zugleich war Philister aber auch die Bezeichnung für Bürger, die sich vor allem in kleinen Universitätsstädten in einem spannungs- und konfliktreichen Verhältnis zu den Studenten befanden (vgl. Bosse 2012). Die Philistersatiren übernehmen die kunstbezogene Konnotation der „Mittelmäßigkeit“ und verallgemeinern sie zum Merkmal einer sozialen Gruppe, der eine ebenso „mittelmäßige“ Position im gesellschaftlichen Gefüge zugewiesen wird: Die in einer ständischen Ordnung mittlere Position der Bürger bzw. des Mittelstands zwischen Adel und einfachem Volk erhält den pejorativen Sinn des „Mediokren“. Die sozialen Unterschiede, die sich in den verschiedenartigen, in Spannung zueinander stehenden Lebenswelten der bürgerlichen Philister und der Studenten um 1800 zeigen, werden in den Philistersatiren der Romantik somit in eine Semantik überführt, die die Kontraste zwischen den Normenhorizonten betont, welche die Lebensführung der beiden Gruppen anleiten. Der studentische Normenhorizont dient dann als Ausgangspunkt für das Ordnungsmodell, in dem den Philistern eine niedrige Position zugewiesen wird. Aus der Gegenüberstellung von Philister und Student übernehmen die Satiren zudem das Motiv eines Generationenkonflikts zwischen Alt und Jung, der geschichtsphilosophisch aufgeladen wird: Das „Philisterhafte“ wird zum Symbol des Veralteten, das Junge und Studentische hingegen zum Kennzeichen des Zukünftigen verallgemeinert. ${ }^{7}$

\footnotetext{
${ }^{6}$ Diese Verachtung entnehmen die Romantiker dem Bewertungsschema, das im Bereich der Kunst seinerzeit bereits vorgezeichnet war - so haben Goethe und Schiller in ihren Distichen in den Xenien (Goethe und Schiller 2004a [1797]) und den Tabulae Votivae (Goethe und Schiller 2004b [1797]) sowie einigen Gedichten bereits das Mittelmäßige und das Philiströse verknüpft.

7 Für eine Diskussion des Konzeptes der Jugend in der Romantik vgl. Oesterle 1997.
} 
In der Philistersatire von Clemens Brentano aus dem Jahre 1811 manifestiert sich diese Funktionsweise auf pointierte Weise, denn in ihr werden bereits bestehende Zuschreibungen an den Philister zusammengeführt und zum Teil überboten. In der als Broschüre veröffentlichten ,[s]cherzhafte[n] Abhandlung“ (Brentano 2013 [1811], S. 123) ,traktiert“ Brentano den Philister in grotesken, sich überschlagenden hyperbolischen Beschreibungen, metapherngestützten Assoziationsketten und unausgeführten Anspielungen ,nach Strich und Faden“ (Oesterle 1992, S. 56). Hier zeigt sich die oben bereits erläutere Funktionsweise der Philistersatire exemplarisch: Brentano erweitert den auf lokalisierbare soziale Gruppierungen verweisenden Konflikt zwischen Studenten und Philistern, indem er ihn in eine Gegenüberstellung überführt, die Lebensweisen und Haltungen in einer weitaus allgemeineren Weise betrifft. „Philister also wurden“, so die dortige Definition,

alle genannt, die keine Studenten waren, und nehmen wir das Wort Student im weitem Sinne eines Studierenden, eines Erkenntnißbegierigen, eines Menschen, der das Haus seines Lebens noch nicht wie eine Schnecke, welche die wahren Hausphilister sind, zugeklebt, eines Menschen, der in der Erforschung des Ewigen, der Wissenschaft, oder Gottes begriffen [...], so stehen die Philister ihm gegenüber, und alle sind Philister, welche keine Studenten in diesem weitern Sinne des Wortes sind. (Brentano 2013 [1811], S. 147)

Die Mittelmäßigkeit des Philisters wird in Brentanos Schrift an Denk- und Lebensführungsweisen verdeutlicht, die dem Publikum seine Minderwertigkeit vor Augen führen. Eine Vielzahl von Praktiken, Überzeugungen und Eigenschaften soll seine Lächerlichkeit bezeugen. In seiner gesamten Lebensführung orientiert am Gewohnten und Gewöhnlichen, so wird suggeriert, suche er Sicherheit in einem strikt geregelten Tagesablauf und Lebensweg. Die Einhaltung sozialer Normen und Konventionen sei das Prinzip seines Ethos, das Geschäft Zentrum seines Lebens. Er strebe nach finanziellem Erfolg, verliere sich dabei jedoch in der Routine, sodass die Geschäftigkeit letztlich zu sinnentleertem Beschäftigtsein verkomme. Diese Lebensweise verweise ihn, eben mit Ausnahme der Erwerbsarbeit, auf den Raum des Privaten, der seiner Kontrolle unterliege und ihm die Abschottung gegen mögliche Irritationen ermögliche (vgl. dazu Engel 2020). Die Sphäre der Öffentlichkeit nehme der Philister vorrangig als den Ort des Geschäfts wahr, der das Einhalten enger Sittlichkeitsregeln verlangt und ihm deshalb durchweg mäßiges Vergnügen bereitet (vgl. dazu auch Novalis 1798, S. 94). Diese Abschottung gegen das Außergewöhnliche ist laut Brentano zugleich Ursache wie Effekt mangelnder Phantasie und resultiert in einer Borniertheit, die sich in der Konzentration auf das Alltägliche und das nahe Umfeld manifestiert. Dagegen werden jene aufgewertet, die sich der Welt zuwenden, sich Herausforderungen stellen, die Erweiterung ihres Horizonts durch Erweiterung ihres Wissens und schließlich eine ungehemmte Entfaltung ihrer Individualität anstreben. ${ }^{8}$ Diese Ideale werden jenen zugeschrieben, die Brentano unter der Bezeichnung „Studierende“ (Brentano 2013 [1811], S. 147) zusammenfasst. Sie kennzeichnet ihm zufolge ein Ethos, der auch die bewusste Überschreitung von Normen und den Willen zur Provokation umfasst.

\footnotetext{
8 Vgl. zum romantischen Konzept der Individualität als Ausdruck von „Einzigartigkeit“ Eberlein 2000.
} 
Verstärkt wird die invektive Valenz dieser Charakterisierung des Philisters durch die Verortung in einer temporalen Ordnung, die den Generationenkonflikt zwischen jungen Studenten und älteren Bürgern verallgemeinert, indem sie ihn als Gegensatz von Vergangenheit und Zukunft deutet. Diese geschichtsphilosophische Dimension, die sich schon im Titel Der Philister vor, in und nach der Geschichte andeutet, zeigt sich in Anleihen aus der christlichen Mythologie. So wird der Philister mehrfach mit Luzifer identifiziert, der sich in einem ewigen Konflikt mit Gott befinde (ebd., S. 132 ff.). In seinem ,diesseitigen“ Dasein wird er allerdings auf eine Existenz als Repräsentant des Vergangenen und des Apologetentums im Künstlerischen reduziert (ebd., S. 148, 166). In der Gegenwart verkörpere er eine Art Zombie-Existenz, wie Brentano plastisch ausführt:

Ein Philister ist ein steifstelliger, steifleinener, oder auch lederner, scheinlebendiger Kerl, der nicht weiß, daß er gestorben ist, und ganz unnöthiger Weise sich länger auf der Welt aufhält; ein Philister ist ein mit allerlei lächerlichen äußerlichen Lebenszeichen behängter umwandelnder Leichenbitterstock seines eigenen innern ewigen Todes. (ebd., S. 128)

Das Tote wird damit zu einem Merkmal des Philisters, das ihn wesenhaft - also essenziell - bestimme. Die Essenz der „Studierenden“ dagegen sei das Leben, sie seien „großen und herrlichen Flüsse[n]“ vergleichbar, die ,,sich aus reinen ursprünglichen fröhlichen Quellen der Gebürge" speisen (ebd., S. 125). Gegenüber der geistigen Stagnation des Philisters wird die permanente Veränderung aufgewertet als eine auf Dauer gestellte Suche mit dem Ziel, das eigene Selbst zu entfalten und den eigenen „Genius“ zu entwickeln.

„Student“ und „Bürger“ sind hier also keine von Personen eingenommenen sozialen Rollen, sondern Chiffren für Lebensweisen und Haltungen. Als Studierende können sich nicht nur jene bezeichnen, die gerade an einer Universität ausgebildet werden, um danach die Rolle zu wechseln und in eine bürgerliche Existenz einzutreten, also ein Amt in Kirche oder Staat auszufüllen. Vielmehr wird die Möglichkeit eröffnet, das Studentendasein als eine dauerhafte Lebensform zu begreifen. Die Herabsetzung des Philisters erweist sich in dieser Weise als ein Desidentifikationsangebot. Seine Lebensweise wird lächerlich gemacht und zugleich insinuiert, man müsse nicht in dieser Weise leben, sondern könne auch anders sein. Damit dient sie auch dem Angriff auf die hegemoniale Hierarchisierung und Bewertung der sozialen Positionen und treibt deren Umwertung voran: Indem die Philister als den Studenten gegenüber inferior dargestellt werden, wird die bestehende gesellschaftliche Hierarchie invertiert.

An den Philistersatiren der Romantik zeigt sich damit die das Spießerverdikt kennzeichnende diskursive Strategie, die die Abwertung des anderen und die Hochbewertung des Eigenen in einem Positionierungsakt verknüpft. In ihren Charakterisierungen wird der Philister einerseits in einer räumlich gedachten sozialen Mitte, andererseits temporal in der Vergangenheit positioniert. Die Lebensweise und Haltung der Studenten wird demgegenüber im gleichen Zug als peripher und avantgardistisch markiert. Die Orientierung auf eine offene Zukunft und die Überzeugung, diese in besserer Weise als die ,mittelmäßigen“ Philister gestalten zu können, ist damit untrennbar mit dieser Invektive verbunden: Wer ihr folgt und auf die Philis- 
ter herabschaut, muss sich zugleich an der zugrundeliegenden Ordnungsvorstellung orientieren und sich selbst als beweglich und zukunftsoffen ansehen. Ironie und Hyperbolie, insbesondere aber das Verlachen der Philister werden als taktische Mittel eingesetzt, um $\mathrm{zu}$ affizieren und $\mathrm{zu}$ provozieren und hegemoniale Verhaltensregeln zu unterminieren.

Die Philisterschmähungen, die die romantischen Satiren zu Beginn des Jahrhunderts entwickeln, bleiben in den folgenden Dekaden virulent und in ihren Funktionen als Desidentifikationsangebot und Herabsetzung einer sozial mittigen Position maßgeblich. Am Ende des Jahrhunderts prägt insbesondere Friedrich Nietzsche mit der invektiven Bezeichnung „Bildungsphilister“ (Nietzsche 1999 [1873], S. 165) eine Variante, die die Herkunft dieses Spießerverdikts aus dem ästhetischen Feld betont. ${ }^{9}$ Seit Mitte des 20. Jahrhunderts ist die Bezeichnung nur noch als bildungssprachlicher Ausdruck bekannt. Ihre Funktion als Desidentifikationsangebot beerbt seit ungefähr dem zweiten Drittel des 19. Jahrhunderts die Bezeichnung Kleinbürger, die in eine neue Deutung der sozialen Ordnung eingebettet ist.

\subsection{Die Kleinbürgerkritik in der Klassentheorie}

Die Kleinbürgerkritik ist eines der wirkmächtigsten Spießerverdikte des 19. Jahrhunderts, das auch das 20. Jahrhundert noch kennt und nutzt (Enzensberger 1982). Sowohl in der Sozialgeschichte als auch in der Soziologie ist das Kleinbürgertum zwar als eine analytische Bezeichnung anerkannt, die sich auf Berufsgruppen sowie Produktions- und Lebensführungsweisen bezieht und die es von anderen sozialen Gruppierungen abgrenzt (vgl. z.B. Haupt und Crossick 1998; Eder 1989). Jedoch scheint gerade der Kleinbürger als Objekt wissenschaftlicher Betrachtung schwierig zu fassen, gilt der häufig ,als Schimpfwort, oft mit ironischem Beigeschmack“ verwendete Begriff doch als ,,[z]u stark mit ideologischem und polemischem Gehalt aufgeladen“ (Franke 1988, S. 9). Unter der hier gewählten Perspektive, die die Schmähung als eine Praxis der Herabsetzung ernst nimmt, wird die Not der Historiker*innen jedoch zu einem Gewinn für Soziolog*innen, wenn die Bezeichnungen Kleinbürger, Spieß(bürg)er und Philister als Kampfmittel in Auseinandersetzungen um die Deutung der sozialen Ordnung in den Blick genommen werden.

Artikuliert wird die Kleinbürgerkritik als Invektive gegen die mittleren Lagen seit Mitte des 19. Jahrhunderts in der Klassentheorie (vgl. dazu Schrage 2020). Die prominentesten und auch differenziertesten Autoren sind hier Karl Marx und Friedrich Engels. Wir konzentrieren unsere folgende Darstellung deshalb auf das Manifest der Kommunistischen Partei von 1848 sowie auf Engels' unvollständig überliefertes Manuskript „Der Status Quo in Deutschland“ von 1847. Mit der Erzeugung einer neuen Variante des Spießerverdikts wird - trotz einiger bemerkenswerter Kontinuitäten zur Philistersatire - eine Diskursverschiebung ersichtlich. Die Kleinbürgerkritik greift nämlich auf eine anders geartete Vorstellung der sozialen Ordnung zurück, sodass

\footnotetext{
9 Dabei ist die Bezeichnung Bildungsphilister ebenfalls bereits für die Romantik dokumentiert - Nietzsche nimmt jedoch die Prägung dieses Kompositums für sich in Anspruch (vgl. Meyer 1963). Man kann die Invektive aufgrund ihres veränderten Bedeutungsgehaltes und ihres Auftauchens in einem völlig veränderten historischen Kontext allerdings durchaus als neue Prägung interpretieren.
} 
die Problematisierung des Kleinbürgers innerhalb eines anderen Bewertungsschemas stattfindet.

Mit dem Übergang vom Philister zum Kleinbürger verändert sich zudem der epistemische Status der invektiven Figur und damit auch ihre Funktion. Die Kleinbürgerkritik folgt zwar der für das Spießerverdikt insgesamt charakteristischen diskursiven Strategie, nutzt allerdings eine andere Taktik als die Philistersatire. In der mit wissenschaftlichem Anspruch auftretenden Klassentheorie wird der Kleinbürger als Vertreter einer Klasse eingeführt, die objektiv bestimmbar sei - dem Kleinbürgertum. Entsprechend dieser objektivierenden Anlage der klassentheoretischen Analyse erscheint das Kleinbürgertum als eine Zwischenklasse in einer prekären Stellung: Sie ,schwebt“" zwischen der aufstrebenden Bourgeoisie und dem entstehenden Proletariat und wird damit in einer mittleren sozialen Lage positioniert (Marx und Engels 1959 [1848], S. 484). Von besonderer Bedeutung für die Herabsetzung des Kleinbürgers in der Klassentheorie ist jedoch, dass dieser Position wiederum jegliche Zukunftsfähigkeit abgesprochen wird, indem sie als Phänomen eines Übergangs gefasst wird. Wie schon der Philister repräsentiert das Kleinbürgertum ein Residuum der sich im Prozess der Veraltung befindlichen Ordnung. Auch der Herabsetzung der Kleinbürger liegt also eine komplexe geschichtsphilosophische Positionierungspraxis zugrunde, die aus einer angemaßten Position zukunftsträchtiger Randständigkeit erfolgt. Diese Positionierung ist die Grundlage für die in verschiedenen Schriften von Marx und Engels aufzufindende, durchaus invektive Charakterisierung des Kleinbürgertums als unselbständige und passive Klasse, laut Engels die ,miserabelste [...], die zu irgendeiner Zeit in die Geschichte hineingepfuscht hat" (Engels 1959 [1847], S. 46). Die objektivierende Klassenanalyse des Kleinbürgertums bereitet den Boden für die Disqualifizierung dieser Klasse und ihrer Vertreter, den Kleinbürgern. Realisiert wird sie in der Abwertung der kleinbürgerlichen Lebensweise und politischen Haltung.

Bekanntermaßen präsentiert das Manifest die Grundzüge dieser komplexen Geschichtsphilosophie in Form eines Narrativs, das die „Geschichte aller bisherigen Gesellschaft“" als ,eine Geschichte von Klassenkämpfen“ modelliert (Marx und Engels 1959 [1848], S. 462). Jede historische gesellschaftliche Formation sei durch eine spezifische Konfliktkonstellation gekennzeichnet, in der sich „Unterdrücker“ und „Unterdrückte“ in einem ,ununterbrochenen, bald versteckten, bald offenen Kampf“ befänden, der schließlich entweder mit einer ,revolutionären Umgestaltung der ganzen Gesellschaft endete oder mit dem gemeinsamen Untergang der kämpfenden Klassen“ (ebd.). Laut der Analyse befinden sich die europäischen Länder zeitgenössisch in einer solchen Phase der tiefgreifenden gesellschaftlichen Restrukturierung, da ,,aus dem Untergang der feudalen Gesellschaft“" die „,moderne bürgerliche Gesellschaft" (ebd., S. 463) hervorgegangen sei, in der sich die Bourgeoisie durchsetzte und das Proletariat sich als die neue unterdrücke Klasse konstituiert. Vor dem Hintergrund dieses Schemas wird ,der Kleinbürgerschaft“ prognostiziert, ,ins Proletariat herabgeschleudert [zu] werden“" und mittel- bis langfristig ,,als selbständiger Teil der modernen Gesellschaft gänzlich [zu] verschwinden“ (ebd., S. 484). Erst dann könne die Konfrontation von Bourgeoisie und Proletariat klar hervortreten und der Kampf des Letzteren sich ganz auf die Abschaffung der kapitalistischen Produktionsweise konzentrieren. 
Vor dem Hintergrund dieses historischen Narrativs, das das zukünftige Verschwinden des Kleinbürgertums antizipiert, nimmt sich die Gegenwart insbesondere in Deutschland als Anomalie aus. Hier nämlich erhebe das Kleinbürgertum Anspruch auf Hegemonie - Engels zufolge ein untrüglicher Ausdruck der deutschen Misere und einer im Vergleich zu Westeuropa „zurückgebliebenen Zivilisationsstufe“ (Engels 1959 [1847], S. 43). Während sich in anderen Ländern im Prozess der Überwindung des feudalen Systems die Bourgeoisie als diejenige Klasse herausbildet habe, die die Vormachtstellung erlangt hat, sei es in Deutschland die „Klasse der Kleinbürger“, die „,nach der Herrschaft strebt“ (ebd., S. 44). Sie sei allerdings zu „schwach“ (ebd., S. 49), ,hilflos[]“ (ebd., S. 48) und „undiszipliniert[]“ (ebd.), um die Macht des noch herrschenden Adels zu brechen.

Grund dafür seien die Lebens- und Produktionsweisen der Kleinbürger, die sich durch Beschränkt- und Borniertheit auszeichneten - also jene Eigenschaften, die wie gesehen bereits den Philistern in den romantischen Satiren attestiert worden waren. In den klassentheoretischen Invektiven zeigt sich nun jedoch eine andere Rahmung, die die Lebensweisen weniger stark als individuell und frei wählbar erscheinen lässt. Statt des Normenhorizonts der individuellen Kleinbürger wird der unterstellte Interessenhorizont in den Fokus gerückt, der ihnen als Klasse gemeinsam sei und sich als bestimmend erweise. So vertreten sie Engels zufolge „Interessen, die nicht über den engsten Lokalkreis hinausreichen“, und haben „,notwendig einen ihren beschränkten Lebensverhältnissen entsprechenden beschränkten Gesichtskreis“ (ebd., S. 53). Daraus ergibt sich die Unfähigkeit des Kleinbürgertums, eine politische Kraft zu werden oder einen Staat zu führen, denn der Kleinbürger als Klassenvertreter habe ,nur insofern Interesse an der allgemeinen Politik seines Landes, als er den Frieden wünscht; sein bornierter Lebenskreis macht ihn unfähig, Relationen von Staat zu Staat zu übersehen" (ebd, S. 56). Angesichts einer sich globalisierenden Wirtschaft sei es nur die Bourgeoisie mit ihren ,umfassenderen Interessen, größerem Besitz und entschiedenerem Mut“ (ebd., S. 44), die Deutschland aus dem „Morast seines Status quo" (ebd., S. 42) führen könne.

Repräsentiert der Kleinbürger also einerseits in sozialstruktureller Hinsicht die mittlere Position zwischen Bourgeoisie und Proletariat, so ist er andererseits Repräsentant einer Übergangsordnung, die - ähnlich der Philisterfigur in den romantischen Satiren - in der Gegenwart bereits ihre Existenzberechtigung verloren hat. Das deutsche Kleinbürgertum, so das Manifest, sei seit dem 16. Jahrhundert in immer neuen Formen in Erscheinung getreten (Marx und Engels 1959 [1848], S. 487). Gleichwohl wird ihm als Klasse jede aktive Rolle in der Gestaltung der Zukunft abgesprochen. Aus dem Anspruch, dass die eigene Sichtweise auf der objektiv richtigen Analyse der Funktionsweise der kapitalistischen Produktionsweise und ihrer historischen Entwicklungen beruht, ergibt sich für die Klassentheoretiker das eindeutige Verdikt, dass die Produktions- und damit Lebensweise des Kleinbürgertums nicht mehr zeitgemäß sei. Es wird damit als ein welthistorischer Anachronismus markiert, dessen Fortleben jedoch weitreichende Konsequenzen hat: Das Kleinbürgertum fungiere eben gerade in Deutschland als Hemmschuh der geschichtlichen Entwicklung. Doch selbst in dieser Funktion könne es nicht einmal mehr den ,Schein historischer Initiative" bewahren, sondern widersetze sich den historisch-gesellschaftlichen Dynamiken „mit dem ganzen Gewicht [seiner] Trägheitskraft“ (Engels 1959 [1847], S. 
47). Das Kleinbürgertum sei damit eine genuin konservative Macht, es „klammert sich an das Bestehende“, so Engels, und befördere die Stagnation der historischen Entwicklung in Deutschland. Ironisch fügt Engels hinzu: „So hat die Kleinbürgerschaft bei ihrer gedrückten gesellschaftlichen und politischen Stellung wenigstens den Trost, die Normalklasse von Deutschland zu sein und allen übrigen Klassen ihre spezifische Gedrücktheit und ihre Nahrungssorgen mitgeteilt zu haben“ (ebd., S. 50 f.). So trage auch das deutsche Bürgertum ,den Stempel bürgerliche[r] Mittelmäßigkeit", nimmt sich gegenüber der Bourgeoisie in den industrialisierten Staaten „winzig“, „unbedeutend“ und „lokalborniert“ aus (ebd., S. 50).

Der Kleinbürger wird damit zu einer genuin politischen Figur gemacht, und zwar gerade weil er sich weigert, auf der Höhe der Zeit zu agieren und sich den politischen Auseinandersetzungen zu stellen, die in den Augen Engels' und Marx' die Agenda bestimmen sollten. Die Kämpfe des Kleinbürgertums um Machterhaltung verhindern demnach die Polarisierung von Bourgeoisie und Proletariat, die im Klassenkampfnarrativ als erstrebenswert erscheint - denn erst die direkte Konfrontation von Bourgeoisie und Proletariat würde ja Marx und Engels zufolge die proletarische Revolution und damit die menschliche Emanzipation herbeiführen. Die Macht der Kleinbürger zu brechen ist mithin ein Schritt, der in der materialistischen Geschichtsauffassung als notwendig erscheint. Die starke Polemik gegen die Klasse der Kleinbürger wird zum integralen Bestandteil der Strategie, die eigene Ordnungsvorstellung durchzusetzen.

Vor dem Hintergrund dieses Narrativs kann deshalb die Zuschreibung einer progressiven, den Fortschritt repräsentierenden Kraft sowohl an die Bourgeoisie als auch an das Proletariat - inklusive der mit ihm verbündeten Kommunisten - vergeben werden. Wiederum wird dabei die deutsche Situation als eine spezifische markiert: Die Bourgeoisie in England und Frankreich befinde sich laut Marx und Engels derzeit auf Seiten des Fortschritts. Allerdings wird auch ihr prophezeit, zur Vertreterin der Stagnation zu werden. Derzeit trage sie jedoch dazu bei, die Institutionen und traditionellen Privilegien des feudalen Systems zu beseitigen und sich als die herrschende Klasse zu behaupten, indem sie ,die heiligen Schauer der frommen Schwärmerei, der ritterlichen Begeisterung, der spießbürgerlichen Wehmut in dem eiskalten Wasser egoistischer Berechnung ertränkt“ und die „festen eingerosteten Verhältnisse" auflöse (Marx und Engels 1959 [1848], S. 464 f.). Sobald sich jedoch ihre Macht konsolidiere, wenn sie ,aufhört, progressiv und revolutionär zu sein, und selber stationär wird“ (Engels 1962 [1845], S. 580), werde die Position der Progressiven und Revolutionäre an die Arbeiterklasse bzw. das Proletariat übergehen. Weil das Bürgertum in Deutschland jedoch vom Kleinbürgertum dominiert werde, wird die Position der zukunftsfähigen Vertreter des Fortschritts hier freigehalten für die eigene Gruppe, die Kommunisten. Sie „,kämpfen“, so Marx und Engels im Manifest, ,für die Erreichung der unmittelbar vorliegenden Zwecke und Interessen der Arbeiterklasse, aber sie vertreten in der gegenwärtigen Bewegung zugleich die Zukunft der Bewegung“ (Marx und Engels 1959 [1848], S. 492). Die von den Romantikern genutzten Gegensatzpaare alt versus jung und tot versus lebendig werden in den Invektiven gegen die Kleinbürger also durch eine neuartige Geschichtsphilosophie begründet, der eine klare Teleologie eignet. Gegenüber der recht konturlosen Zukunftsvision der Romantiker, die ihren eigenen Anspruch auf Zukunftsfähigkeit 
vor allem dadurch anmeldet, dass sie der Erwartung eines festgelegten biographischen Lebensweges widerspricht und dagegen die individuelle Selbstbestimmung setzt, unterliegt der Klassentheorie die kollektive Zukunftsvision einer kommenden Gesellschaftsordnung.

Die Kleinbürgerkritik zeichnet sich gegenüber der Philistersatire somit einerseits durch eine größere Komplexität, einen höheren Theoretisierungsgrad und einen politisch motivierten Mobilisierungsappell aus. Andererseits ist aber auch die Ähnlichkeit in der Funktion erkennbar: Die Herabsetzung des Kleinbürgertums dient der Aufwertung der eigenen Gruppe und generiert ein Bewertungsschema, das Positionen in einer räumlichen Ordnungsdimension mit solchen in einer zeitlichen Ordnungsdimension koppelt, wobei jeweils die Position der Mitte zwischen oben und unten respektive des Übergangs zwischen alt und neu invektiert wird. Die Mitte wird hier also nicht, wie im Liberalismus, als Aktivitätszentrum der Gesellschaft codiert, sondern auf dem zeitlichen Register als Hemmschuh einer Entwicklung und auf dem räumlichen als unentschiedenes juste milieu diskursiviert. Als Innovation gegenüber der romantischen Variante des Spießerverdikts erweist sich die Verknüpfung der sozioökonomischen Situierung mit der den Kleinbürgern zugeschriebenen Haltung zu den historischen Dynamiken. Sie lässt die Intervention in die bestehende, als veraltet und überkommen erscheinende Ordnung als legitim erscheinen, womit der Aufruf zur Desidentifikation mit dieser Klasse seinen spezifischen Sinn erhält. Die diskursiven Praktiken der sozialen Positionierung beziehen sich hier also auf eine sehr differenzierte Vorstellung der sozialen Ordnung, der ein ausgearbeitetes Modell der Sozialstruktur zugrunde liegt, auf dessen Basis Akte der sozialen Positionierung in der Mitte einer Beleidigung gleichkommen können.

Die Kleinbürgerkritik bleibt als Modell der Spießerbeschimpfung bis weit ins 20. Jahrhundert hinein verbreitet. Die Relektüre der Schriften von Marx und Engels in Gegenkulturen wie der Studentenbewegung seit den 1960er-Jahren macht sie relevant in der Alltagskommunikation und dem Feuilleton. Jedoch zeigt sie sich auch in soziologischen Theorien, die Impulse aus der Marx'schen Theorie aufnehmen. Besonders augenfällig ist dabei die Verwendung bei Pierre Bourdieu, dessen Charakterisierung der Kleinbürger in Die feinen Unterschiede eindeutig durch die Klassentheorie Marx' affiziert ist. Der Lebensstil des Kleinbürgers wird wiederholt in einem pejorativen Sinne mit dem „Mittleren“ identifiziert, wie z.B. der ,,mittleren Kunst“ (Bourdieu 1982, S. 110). Ihm werden Eigenschaften wie „Nüchternheit und Maßhalten“ (ebd., S. 297), „Gehemmtheit“ (ebd., S. 331) und „Geschäftigkeit“ (ebd., S. 337) zugeschrieben. Der ,ambitionierte“ Kleinbürger ,versagt“ sich das Leben in der Gegenwart, ,im Hier und Jetzt“, und bildet ,sein Selbstbild um den Gegensatz zwischen Heim und Café" - wobei er wie der Philister das Heim und das Private präferiert - sowie ,zwischen individuellem Wohl und kollektiver Solidarität“ (ebd., S. 297). Wie bei Marx und Engels erweist sich der Kleinbürger also auch bei Bourdieu als borniert und kapriziert auf seinen kleinen Einflussbereich.

\subsection{Antibürgerliche Selbstinszenierung der Boheme}

Als dritte Variante des Spießerverdikts betrachten wir nun die dezidiert antibürgerlichen Invektiven, die im Kontext verschiedener Kreise und Bewegungen der 
Boheme ${ }^{10}$ produziert wurden. Unter der Bezeichnung Boheme als soziokultureller Formation wird hier ein breites Spektrum von literarischen und künstlerischen Strömungen gefasst, die aufgrund ihrer Heterogenität kaum auf einen Nenner zu bringen sind. Helmut Kreuzer, der bereits 1968 eine klassisch gewordene Studie zur Boheme veröffentlichte, rechtfertigt die Rede von der Boheme als soziokultureller Kategorie jedoch damit, dass in ihr ein ,,bestimmter, intentionell unbürgerlicher Stil des Lebens“ praktiziert werde, der mit einer ,gegenbürgerlichen Einstellung“ verbunden sei (Kreuzer 2000 [1968], S. 43). Die Boheme sei historisch gebunden an die bürgerliche Gesellschaft, sie sei ein ,ihr zugehöriges antagonistisches Komplement“ (ebd., S. 45), das sich nur auf Grundlage einer bestimmten gesellschaftlichen Konstellation habe entwickeln können (vgl. auch Bourdieu 1999, S. 83 ff.). Anders als im Falle des Philisters oder des Kleinbürgers kristallisiert sich indes in der Antibürgerlichkeit der Boheme keine Bezeichnung eines invektivierbaren Sozialtypus. Allerdings finden sich beide älteren Typen - neben der vermehrten Verwendung des Spießbürgerbegriffs - in den Schriften der Boheme, sodass Kreuzer in den literarischen Zeugnissen ein „Philisterbild“ (ebd., S. 142) und ein „Bourgeoisbild“ (ebd., S. 146) unterscheidet. Diese sind jedoch nicht kongruent zu den oben vorgestellten romantischen respektive klassentheoretischen Varianten dieser Figuren: Vielmehr sind sie auf die Boheme hin aktualisiert und zeigen deshalb eine weitere Variation des Spießerverdikts an.

So wird die Strategie der Abwertung der Position in der gesellschaftlichen Mitte in den Spießerverdikten der Boheme taktisch durch einen hohen Grad an Selbstinszenierung realisiert, die das Bestreben um die Aufwertung der eigenen Lebensweise stützt. Dies macht den besonderen Charakter dieser Variante aus. Einerseits wird sich hier explizit von der klassentheoretischen Variante abgegrenzt. Andererseits werden Elemente der romantischen Individualitätsrhetorik aufgenommen, diese jedoch angesichts eines veränderten historischen Kontextes aktualisiert und stärker noch als bei den Romantikern als praktizierte Lebenskunst realisiert. Im Folgenden werden diese Bezüge auf die vorangegangenen Varianten des Spießerverdikts und die ihnen korrespondierenden Ordnungsvorstellungen anhand von Diskursbeiträgen Erich Mühsams, der als einer der herausragenden Boheme-Vertreter in Deutschland gilt, sowie Julius Babs vorgestellt, der u.a. in seiner Monographie Die Berliner Boheme (1904) auf die Boheme-Bewegung reflektiert.

Allgemein gesprochen wird die gesellschaftliche Mitte in den Spießerverdikten um 1900 nicht vorrangig als „,mittlere Klasse“ in einem hierarchischen Gefüge verstanden, wie es in der Klassentheorie der Fall ist. Vielmehr nehmen die Bohemiens die bereits durch die Romantiker eingeführte Assoziierung von Mittelmäßigkeit und Konformität auf und überformen sie indem sie die Mitte als ein integriertes Zentrum der Gesellschaft konzipieren von dem ein exkludiertes Außen abgesetzt ist. Darin kommt eine Vorstellung der gesellschaftlichen Mitte zum Ausdruck, die heute noch in politischen Diskursen zu finden ist, die zwischen den Volksparteien als „Parteien der Mitte“ und den davon abgesetzten politischen Rändern unterscheiden (vgl. u.a. Marg 2014).

10 Wir verwenden in diesem Beitrag das Lehnwort Boheme statt des Fremdworts Bohème, um deutlich zu machen, dass wir uns vorrangig auf den deutschsprachigen Diskurs beziehen. 
Wie in den beiden anderen vorgestellten Varianten werden die (Spieß-)Bürger als Angehörige dieser integrierten Mitte abgewertet, indem sie pauschal mit einem Persönlichkeitstypus identifiziert werden, der sich unhinterfragt an den hegemonialen Normen orientiert, den eigenen ökonomischen Interessen folgt und ein sowohl konservatives als auch beschränktes Leitbild eines erfolgreichen Lebens- und Karriereweges propagiert. Wie schon in der bei Brentano sich abzeichnenden Dichotomie von Philister und Studierendem stehen sich bei Bab Spießbürger und Bohemien gegenüber, wobei Bab die wechselseitige Abwertung gleich zu Beginn seiner Abhandlung betont. Ersterer verbringe sein Dasein im „Gehege der Sitten“ und schaue „mit Grauen und Neugier, Neid und Verachtung“ auf Letzteren (Bab 1904, S. 6). Der Bohemien dagegen, dem aufgrund seiner künstlerischen Ambitionen und ,lebensdurstigen“ Antriebe die „Führung einer ,gesellschaftsfähigen“ Existenz verwehrt“ sei, zeige sich in einer permanenten „kriegerisch-hämischen Stimmung gegenüber der Gesellschaft“, die ihn umgebe (ebd., S. 7). Dem Bürger, der sich im „Gehege“ der Konventionen sozusagen eingesperrt findet und aufgrund seiner Konformität als gesellschaftlich integriert gilt, wird der Bohemien als Angehöriger des „Kultur-Zigeunertums“ entgegengestellt, das die ,zentrifugalen Elemente der Menschheit" repräsentiere (ebd., S. 4). Dies seien die Künstler und Intellektuellen, die sich frei von der Beschränktheit der Bürger dem „Lebenswirrsal“ (ebd., S. 10) hingeben und damit die invektive Bezeichnung Zigeuner aufwerten und sich aneignen. ${ }^{11}$ In ähnlicher Weise modelliert Mühsam die Gegenüberstellung von Philistern und „Ausgestoßenen“, insbesondere den Künstlern: „Philistrosität ist die Tendenz, den eigenen sittlichen Horizont als moralischen Schutzkordon um die Menschheit zu legen.“ Dabei sei jedoch nicht ,,die größtmögliche Anpassung an die Gepflogenheit der Mehrzahl“" das vorrangige Problem, sondern „,die eifersüchtige Bewachung des Nebenmenschen“ (Mühsam 1906, S. 4), insbesondere derjenigen, die sich der „gesellschaftliche[n] Nutzarbeit verweigern“ (ebd., S. 6).

Diese Gegenüberstellungen beruhen somit auf der Unterscheidung zwischen einerseits der Zufriedenheit mit dem Bestehenden, Kennzeichen der Lebensweise des Spießbürgers und Philisters, und andererseits dem „Auftrotzen gegen das Gewöhnliche“ (Mühsam 1906, S. 9) - eine Praktik, die „einer neuen Kultur die Wege“ weisen könne (ebd., S. 10) und damit als zukunftsfähig markiert wird. In den Selbstbeschreibungen der Boheme erscheinen häufig die Positionen, die als soziale Unterschicht gelten und deshalb aus der ,guten Gesellschaft“ exkludiert sind, mit der eigenen verwandt. Nicht das Merkmal der Armut, sondern die Weigerung, sich den bürgerlichen Normen zu unterwerfen, wird insbesondere von Mühsam als Gemeinsamkeit konstatiert. Bohemiens, so Mühsam, zeigten fast immer ,ein inniges Solidaritätsgefühl zum fünften Stande, zum Lumpenproletariat“" (ebd., S. 10).

In der Refiguration des Positionsgefüges wird deutlich, dass diese Variante des Spießerverdikts die klassentheoretische invertiert: Zum einen wird das Proletariat als organisierte Arbeiterschaft, die für die Klassentheorie positiv besetzt ist, nun

\footnotetext{
11 In diesen Aneignungsprozessen wird wenig auf die spezifisch rassistischen Elemente der Zigeunerbezeichnung reflektiert, sondern die marginalisierte Existenz dieser ethnisierten Bevölkerungsgruppe als Freiheit idealisiert. Für eine ausführlichere Betrachtung der Immigration des französischen bohémiens, bohémes und bohême in die deutsche Boheme-Subkultur vgl. Kreuzer 2000 [1968], S. 1 ff.
} 
selbst in einer als problematisch erscheinenden mittleren Position verortet. Das Proletariat stehe um 1900, so Mühsam, ,in der Mitte zwischen Bourgeoisie und den Tschandala, auf deren Seite nur noch die unorganisierten Gruppen kämpfen: Verbrecher, Landstreicher, Huren und Künstler“, die „Ausgestoßenen“ (ebd., S. 5 f.). Die etablierte Arbeiterbewegung habe ,durch die Ausschaltung des individuellen Temperaments des Einzelnen die revolutionäre Kernidee des gewerkschaftlichen Kampfes verwischt“, das Proletariat sei durch die ,zukunftsstaatsbesessene Sozialdemokratie [...] dem Klassenkampf völlig entfremdet" worden (Mühsam 1906, S. 5). Zum anderen stehen der positive Bezug auf das Lumpenproletariat und die Selbstbezeichnung als Boheme der Abwertung dieser Gruppen bei Marx diametral entgegen. In seiner Polemik Der achtzehnte Brumaire des Louis Bonaparte hatte dieser noch der ,ganze[n] unbestimmte[n], aufgelöste[n], hin- und hergeworfene[n] Masse, die die Franzosen la bohème nennen“ (Marx 1960 [1852], S. 161), die Rolle der lächerlichen und moralisch fragwürdigen Unterstützer des Louis Bonaparte zugewiesen und dessen ,langes abenteuerndes Vagabundenleben“ (ebd., S. 159) als Vorbereitung auf seine eigene Rolle als „Chef“ eben dieses „Lumpenproletariats“ interpretiert (ebd., S. 161; Hervorh. weggel.). Letzteres entzieht sich bei Marx konstitutiv der politischen Festlegung und steht als soziale Kategorie quer zu dem oben rekonstruierten klassentheoretischen Ordnungsmodell. Es wird als persona non grata der weltgeschichtlichen „Komödie“ (ebd., S. 161), die sich im Interregnum des zweiten Kaiserreichs ereignet, verächtlich gemacht. Die lumpenproletarischen Bohemiens fügen sich somit nicht reibungslos in das Klassengefüge ein: Sie repräsentieren den Rest, der nach der Einordnung übrigbleibt. Durchaus invektiv bezeichnet Marx sie als „Auswurf, Abfall, Abhub aller Klassen“ (ebd.). ${ }^{12}$

Während das Lumpenproletariat aber bei Marx mit dem Vorwurf politischer Positionslosigkeit belegt wird (vgl. auch Marx und Engels 1959 [1848], S. 472), gilt dieser angebliche Mangel in den Beiträgen der Boheme als Auszeichnung: Das vordem stigmatisierte Vagabundische wird aufgewertet. Wie schon in Brentanos Lob der Studenten bildet Mobilität in geistiger und räumlicher Hinsicht ein tragendes Motiv des Selbstverständnisses. Codiert wird diese Beweglichkeit in den Topoi des Abenteuers, einer Suchbewegung sowie der permanenten Selbstreflexion und -entwicklung. Die Kennzeichen der „Verbürgerlichung“(Bab 1904, S. 36), darunter vor allem die Heirat, das Sich-Niederlassen an einem dauerhaften Wohnsitz und die Aufnahme eines existenzsichernden Berufs, werden hingegen perhorresziert.

Damit einher geht eine erneute Aufwertung der Individualität als Instanz der Freiheit und des Nonkonformen, die die Romantik bereits vorzeichnete und die in der Boheme ganz buchstäblich in die Praxis umgesetzt wird. So stellt in Julius Babs klassisch gewordener Schrift über die Boheme die Beschreibung eines Autors als „Heimatloser“ (Bab 1904, S. 22) und „Gesellschaftsfeind“ (ebd., S. 11), der seine Individualität in vollem Umfang realisiert, das höchste Lob dar. Ein theoretisches Fundament findet diese Haltung in Max Stirners einflussreicher Schrift Der Einzige und sein Eigentum, die eine Lehre des „Egoismus“ enthält und ein mit dem Marxismus konkurrierendes Ideal menschlicher Emanzipation zeichnet (Stirner 2016 [1844]). Auch Friedrich Nietzsche hat sich in Teilen der Boheme-Bewegung als

12 Vgl. zu dieser „Müllmetaphorik“ bei Marx und anderen auch Farzin 2016. 
Referenz für einen solchen emphatischen Begriff des Individualismus etabliert, der die eigene Lebensweise und Haltung ebenso wie die Invektiven gegen Spießbürger und „Bildungsphilister“ (Nietzsche 1999 [1873], S. 165) legitimiert.

Diese die romantischen Ideen aufnehmende Hinwendung zur Individualität zeigt sich auch in der diskursiven Thematisierung der besonderen Lebensführung der Bohemiens, in der die Unterminierung bürgerlicher Normen wie Sparsamkeit, Ruhe, Ordnung und Mäßigung in den Alltagspraktiken ostentativ inszeniert wird. Diese Normen werden verbal und insbesondere anhand der ihnen entsprechenden künstlerischen, meist literarischen Produkte ins Lächerliche gezogen. Darüber hinaus wirken jedoch vor allem die Beschreibungen der eigenen Praktiken im buchstäblichen Sinne stilbildend. Die Bohemiens bringen ihre Verachtung für die bürgerlichen Konventionen durch ihre abgerissene Kleidung, ihre Ablehnung der entfremdeten Lohnarbeit oder des bürgerlichen Unternehmertums, durch das „Pumpen“ als Praxis des Gelderwerbs, die Idealisierung des Hedonismus und die Hingabe an Rausch und Depression gleichermaßen zum Ausdruck (vgl. u.a. Mühsam 1978 [1903]). Gegenüber dem Vernunftideal und dem Mäßigungsimperativ der bürgerlichen Kultur wird die Durchbrechung von Routinen zum Gebot, die im Alltag und in literarischen Werken als gesellige Zusammenkunft im öffentlichen Raum - also gerade nicht in der das (Klein-)Bürgerliche symbolisierenden Privatheit - inszeniert wird und zwischen den Extremen der Selbstisolierung und des konventionensprengenden Feierns mit Fremden oszilliert. Mit diesen Praktiken wird das Leben als Kunstwerk gestaltet (vgl. Magerski 2015, S. 134ff.).

Hinsichtlich der temporalen Ordnung fällt an der antibürgerlichen Haltung der Boheme nun auf, dass der emphatische Fortschrittsbegriff, der die klassentheoretische Kleinbürgerinvektive antreibt, zugunsten einer Hochbewertung des Dynamischen als Eigenwert in den Hintergrund verschoben wird. Die Gegenwart wird nicht als Grenzzone zwischen Vergangenheit und Zukunft konzipiert, sondern als etwas Momenthaftes verabsolutiert, worin die konsequente Ablehnung jeder Stagnation zum Ausdruck kommt. Ein Bohemien ist einer, der „,mit dem Augenblick Fangball spielt“ (Mühsam 1906, S. 9). Bei Marx und Engels wurde die Stagnation, wie gesehen, als eine negativ bewertete Position im Modell des historischen Prozesses durch das Kleinbürgertum und seine Verhaftung in der historisch überkommenen Ordnung repräsentiert. Ähnliches zeigt sich in der Aussage Babs, der den Philister als jenen bestimmt, der ,zu jedem Augenblicke zu sagen bereit ist: verweile doch, du bist ja ausgezeichnet“" (Bab 1921 [1914], S. 125), und sich so mit dem Bestehenden zufrieden zeigt.

Einerseits teilen die Bohemiens also mit der Kleinbürgerkritik die Verachtung des Stillstandes. Andererseits wird aber nun auch die auf kollektive Ziele ausgerichtete geschichtsphilosophische Zukunftsorientierung als philiströs, als eine zu überwindene Unterwerfung unter ein gesellschaftliches Zeitregime präsentiert, wie sich in der oben dargestellten Abwertung der Sozialdemokratie durch Mühsam zeigt. Die Organisierung des Proletariats habe, so seine Beobachtung, zu einer Bürokratisierung geführt, die sich nicht mehr wesentlich von den bereits älteren Institutionen des Staats und der Kirche unterscheide. Eine neue Kultur, für die die „AusgestoBenen“ als Avantgarde fungieren, führe laut Mühsam nur ,über das Grab“ dieser verfestigten und in ihrer Entwicklung stagnierenden Institutionen (Mühsam 1906, 
S. 5). Er referenziert damit das Motiv und den Gegensatz von Leben und Tod, den Brentano in seiner Philistersatire so stark betonte. Der Begriff „Fortschrittsphilister“ (Heym 1914, S. 593) erscheint somit unter den Bohemiens nicht mehr als ein in sich widersprüchliches Kompositum.

In den Spießerverdikten der Boheme zeigt sich mithin ein Ordnungsmodell, das wie seine Vorgänger die Positionierung in der Mitte zur Herabsetzung der bürgerlichen Lebensweisen verwendet und zugleich die eigene aufwertet. Gegenüber der Klassentheorie kann von einer Generalisierungstendenz gesprochen werden, die die Unterscheidung der Klassen des Kleinbürgers und der Bourgeoisie verwischt. Unter dem Schlagwort des Bürgerlichen bzw. Spießbürgerlichen wird, ähnlich wie in der Philistersatire der Romantik, die Abwertung mit Hilfe der Zuschreibungen von Konventionalität und Konformität sowie Mediokrität vorgenommen. Die Überschreitung der philiströsen Beschränktheit und ihrer normativen Grenzen ist Zeichen einer superioren Haltung, die diskursiv verbreitet wird. Eine klare oder gar kollektive Zukunftsvision entwickelt die Boheme dabei allerdings ebenso wie die Romantik kaum. Der starke Imperativ der Selbstexpression ist verbunden mit einer Hypostasierung des Momentanen und des Transitorischen, die in der Hochbewertung räumlicher und geistiger Mobilität zum Ausdruck kommt. Das steht dabei zwar der Prämierung des Kollektiven entgegen, wie sie der Klassentheorie zugrunde lag. Und dennoch beerbt das Spießerverdikt der Boheme insofern die klassentheoretische Variante, als hier die gesellschaftliche Mitte viel deutlicher als ein die Emanzipation verhindernder Ort der Macht thematisiert wird als dies in der fast noch spielerischen romantischen Philistersatire der Fall war.

In seiner bereits angeführten Studie hat Kreuzer die Nähe der Bewegungen der Subkulturen der 1960er-Jahre zur Boheme betont. Noch vor der Politisierung der Gegenkultur veröffentlicht, stehen ihm dabei v.a. die US-amerikanischen Beatniks und Hippies, die auch in Europa Fuß fassten, sowie die „Provos“ vor Augen (Kreuzer 2000 [1968], S. 20 ff.). In ihnen sammeln sich wiederum Nonkonforme, die ihre Ablehnung bürgerlicher Werte in literarischen Produkten, insbesondere aber in ihrer Performanz öffentlich inszenieren - nun jedoch erneut vor dem Hintergrund einer völlig anderen historischen Situation. Hinzuzufügen wären dieser Sammlung insbesondere im deutschen Kontext die Gammler, um die sich Mitte der 1960er-Jahre eine Debatte entspinnt, die wie in der Boheme einen Schwerpunkt in der Thematik der Ablehnung von ,gesellschaftlicher Nutzarbeit“ entfaltet. Christine Magerski (2015) hat diesen Gedanken aufgenommen und weitergeführt: Sie entdeckt in der Boheme einen „Prototyp der Moderne“, der mit seiner Lebenskunst - inklusive der ihr inhärenten Ambivalenzen - einen Lebensstil entworfen habe, der bis in die Ära der ,flüchtigen Moderne“ (Bauman) Wirkkraft entfalte.

\section{Fazit}

In unserer Analyse der drei Varianten des Spießerverdikts im 19. Jahrhundert haben wir gezeigt, dass das Spießerverdikt als ein kommunikatives Muster fungiert, das Gruppen in mittleren sozialen Lagen herabsetzt und dabei zugleich in Auseinandersetzungen um die Deutung der bestehenden sozialen Ordnung als Kampf- 
mittel eingesetzt wird. Seine drei betrachteten Varianten entstehen im Laufe des 19. Jahrhunderts durch die Modifikation, Anreicherung oder Überschreibung der jeweils vorhergehenden Variante, womit um 1900 eine komplexe Semantik des Spießers etabliert ist: die Philistersatire, die Kleinbürgerkritik und die antibürgerliche Selbstinszenierung der Boheme. Diese drei aufeinanderfolgenden Varianten erzeugen im Laufe des 19. Jahrhunderts ein Reservoir an Praktiken der Herabsetzung jener sozialen Gruppen, die die soziale Mitte repräsentieren. Die Verdikte fungieren dabei als Desidentifikationsangebote, wobei sie sich jeweils verschiedener Mittel bedienen. Während Verspottung und Verlachen als die zentralen Überzeugungsmittel der romantischen Philistersatire auszumachen sind, wird die Minderwertigkeit der Mitte in der Kleinbürgerkritik als Ergebnis einer wissenschaftlich-objektiven Analyse dargestellt. Die Taktik der Bohemiens schließlich besteht in der Inszenierung der eigenen Außergewöhnlichkeit und darin, das Spießbürgertum als Masse von Bürgerlichen unterschiedlicher Couleur zu homogenisieren und sich pauschal davon abzugrenzen.

In der Zusammenschau der Varianten lässt sich der diskursive Wandel der Ordnungsvorstellungen, die das Spießerverdikt aufruft und mitgestaltet, in seinen Kontinuitäten und Brüchen nachzeichnen: ${ }^{13}$ Die jeweiligen Positionen der Mitte werden vor dem Hintergrund verschiedener Parameter gebildet, die die jeweiligen Ordnungsvorstellungen markieren, dabei jedoch auch bereits bestehende Varianten reflektieren und modifizierend aufnehmen. In der Philistersatire wird das konfliktreiche soziale Verhältnis zwischen Studenten und Stadtbürgern genutzt, indem die beiden Bezeichnungen von feststehenden gesellschaftlichen Rollen zu frei wählbaren Lebensweisen umgedeutet werden. So kann der unterstellte Konformismus der Majorität dieser als Prinzip ihrer Lebensführung zugeschrieben und sie damit zugleich abgewertet werden. In der Kleinbürgerkritik wird demgegenüber auf Grundlage der Klassentheorie von Engels und Marx die Mitte als Position in einem hierarchisierten Positionengefüge konstruiert, die aufgrund ihrer objektiven Funktion im ökonomisch-historischen Prozess als minderwertig gilt. Plausibilisiert wird dies vor dem Hintergrund einer gewichtigen Geschichtsphilosophie, in der die historische Dynamik im Modus des Fortschritts konzeptualisiert wird. Sozialer Wandel wird in der klassentheoretischen Vorstellung sozialer Ordnung bereits insofern reflektiert, als in ihr drei aufeinanderfolgende Ordnungen postuliert werden: die alte Feudalordnung, die Ordnung der bürgerlichen Gesellschaft sowie eine zukünftige Ordnung, die sich durch die freie Assoziation von Individuen auszeichnet. Deshalb werden in der Klassentheorie unterschiedliche Gruppierungen als Repräsentantinnen des Fortschritts bzw. der Progressivität benannt, die jedoch mit dem Umbruch zur jeweils folgenden Ordnung zu Repräsentantinnen der Stagnation werden: Das Bürgertum bzw. die Bourgeoisie ist in der alten Ordnung eine treibende Kraft für den Ordnungswandel, für die Herbeiführung der menschlichen Emanzipation werde jedoch das Proletariat verantwortlich

\footnotetext{
13 Anders als Boltanski und Chiapello (2003) es in ihrer schematischen Gegenüberstellung von Sozialkritik der Klassentheorie und Künstlerkritik der Boheme suggerieren, kann mit Hilfe der Analyse der Spießerverdikte herausgearbeitet werden, dass und inwiefern sich diese beiden Kritikformen historisch wechselseitig bedingen.
} 
sein. Daraus ergibt sich ein Potenzial für komplexe, theoretisch unterlegte Praktiken der Herabsetzung.

In der Boheme schließlich wird dieser emphatische Fortschrittsbegriff selbst einer veralteten und „verknöcherten“ Haltung zugeordnet und damit einer Abwertung unterworfen. Die ,progressive“ Haltung gilt der Boheme als ebenso anfällig für bornierte und mittelmäßige Lebensweisen wie jene der Spießbürger, die es sich darin bequem machen, den Konventionen zu folgen. Zukunftsfähig seien demgegenüber jene, die in der Lage sind, sich jeder Beschränkung durch Normen und Dogmen zu entziehen, um unter widrigen Umständen ihre eigene Freiheit in größtmöglichem Umfang zu realisieren, anstatt sich der Illusion einer kommenden Revolution hinzugeben. Die abwertende Fremdpositionierung der Philister, Klein- und Spießbürger in der gesellschaftlichen Mitte wird, so zeigt sich in allen Varianten, flankiert durch temporale Indexierungen dieser Position. Während dem rückwärtsgewandtimmobilen Philister die dynamische Zukunftsoffenheit der „Musensöhne“ gegenübergestellt wird, erscheinen die Kleinbürger als Residuum einer Vergangenheit, die sich dem objektiven Geschichtsverlauf widersetzen, den seine Kritiker erkennen und vertreten; die Boheme zeichnet demgegenüber auch den Fortschrittsglauben als eine zu überwindende soziale Norm und setzt ihm einen radikal gegenwartsorientierten Individualismus entgegen, der mit dem (bürgerlichen) Zeitmodell bricht.

Bestimmend für die Spießerverdikte ist also, dass die soziale Mitte zugleich für die zu überwindende soziale Ordnung steht. Der temporale Index der Verdikte zeigt sich auch in zeitlichen Metaphern. So werden die Invektierten diskursiv als Verkörperungen des Toten, der Stagnation und der Unterwerfung unter das herrschende Zeitregime markiert. Die Selbstidentifikation der Invektierenden hingegen wird assoziiert mit Topoi der Dynamik und Entgrenzung: Als Studierender vertritt man das interessante Leben, das die Schranken des Konformen transzendiert, um zu Neuem zu schreiten, als Kommunist die politische und gesellschaftliche Revolution und als Bohemien die Kunst, im Moment zu leben und damit die spießbürgerlichen Erwartungen der Zukunftsplanung zu konterkarieren. Die Zuweisung dieser komplementären Merkmale von Lebensweisen und Normhorizonten an spezifische Positionen in zeitlich und räumlich vorgestellten Ordnungen ist die zentrale diskursive Strategie, mit der das Spießerverdikt operiert. Insofern erschöpfen sich die Spießerverdikte nicht in den Positionierungsakten, die konträre Selbst- und Fremdzuschreibungen beinhalten. Vielmehr lässt sich anhand ihrer Rekonstruktion zeigen, dass ihnen als Mittel der kritischen Reflexion auf das Bestehende bereits eine Vorstellung des sozialen Wandels inhärent ist. Sie verweisen auf Verlaufsmodelle des Sozialen, die sie in kommunikativer Praxis verbreiten, womit sie selbst als Faktor des Wandels wirksam werden.

Derartige praktische Bewertungsakte erhalten in den klassischen Konzeptualisierungen sozialen Wandels, wie sie sich in der Mehrzahl der soziologischen Modernisierungstheorien finden, keine besondere Aufmerksamkeit. Ihre Rekonstruktion erweist sich jedoch als eine Chance, die Funktionsweise gesellschaftlichen Wandels besser zu verstehen indem spezifisch danach gefragt wird, inwiefern invektive Kommunikationsakte als Elemente von Gegendiskursen in die (Re-)Produktion und die Erneuerung von Ordnungsmodellen eingebunden sind. Denn die hier behandelten Varianten des Spießerverdikts entwerfen, wie gesehen, in der Herabsetzung von 
Vertretern der herrschenden Ordnung selbst alternative Ordnungsvorstellungen und katalysieren durch ihre Desidentifikationsangebote den sozialen Wandel, den sie beschwören. Indem die in den Verdikten mitlaufenden Heroisierungen von jugendlicher Dissidenz, revolutionärem Aktivismus und künstlerischer Unabhängigkeit dazu auffordern, die herkömmliche Wertung von richtiger und falscher Lebensführung zu invertieren, reorganisieren sie darüber hinaus das Gefüge der damit verbundenen sozialen Positionen, sodass das soziale Außen oder auch Unten neu bestimmt und zu einem legitimen und unter Umständen gar positiv konnotierten Ort wird, von dem aus gesprochen und gehandelt werden kann oder - normativ gewendet - soll. Dabei sind die Spießerverdikte des 19. Jahrhunderts einerseits selbst Zeugnisse intensiver Auseinandersetzungen um soziale Positionen und Ordnungsvorstellungen, sie stellen aber andererseits auch Deutungsmuster zur Verfügung, die bis heute immer wieder aufgegriffen werden und insgesamt betrachtet zur Entstehung sozialer Rollenmodelle und Milieus beitragen.

Die Genealogie des Spießerverdikts ließe sich ohne Probleme bis in die Gegenwart verlängern. So lassen sich in den Gegen- und Alternativkulturen, die sich seit den 1960er-Jahren etablierten, erneute Aktualisierungen, Anreicherungen und Überschreibungen der romantischen Philistersatire, der Kleinbürgerkritik und der antibürgerlichen Selbstinszenierungen der Boheme finden, die vor dem Hintergrund veränderter historischer Bedingungen bestehende Ordnungsmodelle ver- und neue entwerfen. Die abfällige Rede vom „Establishment“, das in den 1960er- und 1970erJahren als Bezeichnung der spießigen Mehrheitsgesellschaft und Mitte verwendet wurde, ist in das kollektive Alltagswissen eingegangen und wird in jüngster Zeit unter - diesmal politisch invertierten - Vorzeichen wiederbelebt. Daran zeigt sich, dass das kommunikative Muster des Spießerverdikts nicht politisch-ideologisch festgelegt ist, sondern als eine kulturelle Logik, die das Neue hoch bewertet, von höchst unterschiedlichen Akteuren zur Herabsetzung des Bestehenden herangezogen werden kann. So ist in rechtspopulistischen Diskursen die Invektive gegen „Gutmenschen“ ebenfalls als ein Spießerverdikt zu verstehen, das auf einer Vorstellung der sozialen Ordnung beruht, in der die sogenannten Alt-68er die Repräsentanten der aktuellen Mitte sind, deren Normen und Praktiken, z.B. im Umgang mit Migrant*innen, als unzeitgemäß und als Gefahr für die Zukunft Deutschlands markiert werden. Die eigene, angeblich aus einer (marginalisierten) sozialen Peripherie kommende Perspektive wird dagegen als Widerstand gegen einen Konformitätsdruck inszeniert. Und auch in den zu Beginn genannten Figuren des „Bionade-Biedermeiers“, des ,Alternativspießers“, des „Wutbürgers“ und des „Corona-Spießers“ zeigen sich Konflikte um die richtige und zukunftsfähige Lebensweise, den Wert von (nicht-)konformem Verhalten sowie die ihnen inhärenten Aufrufe zur Erhaltung der bestehenden sozialen Ordnung oder zu ihrer Veränderung, handelt es sich also um Varianten des Spießerverdikts, die im Modus des Invektiven auf sozialen Wandel reflektieren und dazu beitragen, ihn weiterzutreiben.

Danksagung Wir danken Anne-Kathrin Hoklas, Mirjam Gräbner, Felix Schilk sowie den anonymen Gutachtenden und der Redaktion für wertvolle und anregende Rückmeldungen zu unserem Beitrag.

Funding Open Access funding enabled and organized by Projekt DEAL. 
Open Access Dieser Artikel wird unter der Creative Commons Namensnennung 4.0 International Lizenz veröffentlicht, welche die Nutzung, Vervielfältigung, Bearbeitung, Verbreitung und Wiedergabe in jeglichem Medium und Format erlaubt, sofern Sie den/die ursprünglichen Autor(en) und die Quelle ordnungsgemäß nennen, einen Link zur Creative Commons Lizenz beifügen und angeben, ob Änderungen vorgenommen wurden.

Die in diesem Artikel enthaltenen Bilder und sonstiges Drittmaterial unterliegen ebenfalls der genannten Creative Commons Lizenz, sofern sich aus der Abbildungslegende nichts anderes ergibt. Sofern das betreffende Material nicht unter der genannten Creative Commons Lizenz steht und die betreffende Handlung nicht nach gesetzlichen Vorschriften erlaubt ist, ist für die oben aufgeführten Weiterverwendungen des Materials die Einwilligung des jeweiligen Rechteinhabers einzuholen.

Weitere Details zur Lizenz entnehmen Sie bitte der Lizenzinformation auf http://creativecommons.org/ licenses/by/4.0/deed.de.

\section{Literatur}

Bab, J. (1904). Die Berliner Boheme. Berlin: Hermann Seemann Nachfolger.

Bab, J. (1921) [1914]. Fortinbras oder Der Kampf des 19. Jahrhunderts mit dem Geiste der Romantik. 3. Aufl. Berlin: Oesterheld \& Co.

Boltanski, L., \& Chiapello, È. (2003). Der neue Geist des Kapitalismus. Konstanz: UVK.

Bonacker, T. (Hrsg.). (2008). Sozialwissenschaftliche Konflikttheorien. Eine Einführung. Wiesbaden: VS Verlag für Sozialwissenschaften.

Bonacker, T., \& Reckwitz, A. (2007). Das Problem der Moderne: Modernisierungstheorien und Kulturtheorien. In T. Bonacker \& A. Reckwitz (Hrsg.), Kulturen der Moderne. Soziologische Perspektiven der Gegenwart (S. 7-18). Frankfurt a. M.: Campus.

Bosaňcić, S., \& Keller, R. (Hrsg.). (2016). Perspektiven wissenssoziologischer Diskursforschung. Wiesbaden: Springer VS.

Bosse, H. (2012). Musensohn und Philister. Zur Geschichte einer Unterscheidung. In R. Bunia, T. Dembeck, \& G. Stanitzek (Hrsg.), Philister. Problemgeschichte einer Sozialfigur der neueren deutschen Literatur (S. 55-100). Berlin: Akademie.

Bourdieu, P. (1982). Die feinen Unterschiede. Kritik der gesellschaftlichen Urteilskraft. Frankfurt a. M.: Suhrkamp.

Bourdieu, P. (1998). Praktische Vernunft. Zur Theorie des Handelns. Frankfurt a. M.: Suhrkamp.

Bourdieu, P. (1999). Die Regeln der Kunst. Genese und Struktur des literarischen Feldes. Frankfurt a. M.: Suhrkamp.

Brentano, C. (2013). Der Philister vor, in und nach der Geschichte. In M. Bergengruen, W. Bunzel, R. Moering, S. Nienhaus, C. Sauer \& H. Schultz (Hrsg.), Texte der deutschen Tischgesellschaft. Bd. 21.1. (S. 122-184). Stuttgart: Kohlhammer.

Bröckling, U. (2007). Das unternehmerische Selbst. Soziologie einer Subjektivierungsform. Frankfurt a. M.: Suhrkamp.

Bührmann, A., \& Schneider, W. (2013). Vom „discursive turn“ zum „dispositive turn“? Folgerungen, Herausforderungen und Perspektiven für die Forschungspraxis. In J. C. Wengler, B. Hoffarth, \& L. Kumiega (Hrsg.), Verortungen des Dispositiv-Begriffs. Analytische Einsätze zu Raum, Bildung, Politik (S. 21-36). Wiesbaden: Springer VS.

Bunia, R., Dembeck, T., \& Stanitzek, G. (2012). Elemente einer Literatur- und Kulturgeschichte des Philisters. Einleitung. In R. Bunia, T. Dembeck \& G. Stanitzek (Hrsg.), Philister. Problemgeschichte einer Sozialfigur der neueren deutschen Literatur (S. 13-54). Berlin: Akademie.

Eberlein, U. (2000). Einzigartigkeit. Das romantische Individualitätskonzept der Moderne. Frankfurt a. M.: Campus.

Eder, K. (1989). Jenseits der nivellierten Mittelstandsgesellschaft. Das Kleinbürgertum als Schlüssel einer Klassenanalyse in fortgeschrittenen Industriegesellschaften. In K. Eder (Hrsg.), Klassenlage, Lebensstil und kulturelle Praxis. Beiträge zur Auseinandersetzung mit Pierre Bourdieus Klassentheorie (S. 341-394). Frankfurt a. M.: Suhrkamp.

Ellerbrock, D., Koch, L., Müller-Mall, S., Münkler, M., Scharloth, J., Schrage, D., \& Schwerhoff, G. (2017). Invektivität - Perspektiven eines neuen Forschungsprogramms in den Kultur- und Sozialwissenschaften. Kulturwissenschaftliche Zeitschrift, 2(1), 2-24. 
Engel, S. (2020). Im Spiegel der Öffentlichkeit. Philister und Kleinbürger als invektive Sozialfiguren des Privaten. In J.-P. Kruse \& S. Müller-Mall (Hrsg.), Digitale Transformationen der Öffentlichkeit (S. 207-228). Weilerswist: Velbrück.

Engel, S., \& Schrage, D. (2021). Das Spießerverdikt. Invektiven gegen die Mittelmäßigkeit der Mitte im 19. Jahrhundert. Bielefeld: Transcript. (im Erscheinen)

Engels, F. (1959) [1847]. Der Status quo in Deutschland. In K. Marx \& F. Engels, Werke (MEW). Bd. 4 (S. 40-57). Berlin: Dietz.

Engels, F. (1962) [1845]. Deutsche Zustände I-III. In K. Marx \& F. Engels, Werke (MEW). Bd. 2 (S. 564-584). Berlin: Dietz.

Enzensberger, H. M. (1982). Von der Unaufhaltsamkeit des Kleinbürgertums. Eine soziologische Grille. In H. M. Enzensberger, Politische Brosamen (S. 197-206). Frankfurt a. M.: Suhrkamp.

Farzin, S. (2016). „Der Auswurf, Abfall, Abhub aller Klassen“ - Müllmetaphorik und Ungleichheit in der soziologischen Zeitdiagnose. In M. Junge (Hrsg.), Metaphern soziologischer Zeitdiagnosen (S. 143-159). Wiesbaden: Springer VS.

Feldenkirchen, M. (2020). Wir Corona-Spießer. Der Spiegel vom 26. Mai 2020. URL: https://www. spiegel.de/politik/deutschland/wir-corona-spiesser-a-00000000-0002-0001-0000-000171037295. Zugegriffen: April 2021.

Fischer, P. (2007). Mitte, Maß und Mäßigkeit. Zur Idee und Relevanz eines gesellschaftlichen Mittebezuges. Hamburg: Dr. Kovac.

Foucault, M. (1981). Archäologie des Wissens. Frankfurt a. M.: Suhrkamp.

Franke, B. (1988). Die Kleinbürger. Begriff, Ideologie, Politik. Frankfurt a. M.: Campus.

Füssel, M., \& Neu, T. (2010). Doing Discourse. In A. Landwehr (Hrsg.), Diskursiver Wandel (S. 213-235). Wiesbaden: VS Verlag für Sozialwissenschaften.

Gelhard, A., Alkemeyer, T., \& Ricken, N. (2013). Techniken der Subjektivierung. Paderborn: Wilhelm Fink.

Gertenbach, L. (2008). Geschichte, Zeit und sozialer Wandel. Konturen eines poststrukturalistischen Geschichtsdenkens. In A. Reckwitz \& S. Moebius (Hrsg.), Poststrukturalistische Sozialwissenschaften (S. 208-226). Frankfurt a. M.: Suhrkamp.

Goethe, J. W., \& Schiller, F. (2004a) [1797]. Tabulae Votivae. In F. Schiller, Sämtliche Werke. Bd. 1 (S. 291-305). München: DTV.

Goethe, J. W., \& Schiller, F. (2004b) [1797]. Xenien. In F. Schiller, Sämtliche Werke. Bd. 1 (S. 309-360). München: DTV.

Hahn, H. (2009). „Ihr Spießer! “ Eine Analyse des Spießertums. Berlin: Pro Business.

Haupt, H.-G., \& Crossick, G. (1998). Die Kleinbürger. Eine europäische Sozialgeschichte des 19. Jahrhunderts. München: Beck.

Heym, G. (1914). Der Philister. März, 8(2), 587-596.

Hume, D. (2003) [1752]. Of refinement in the arts. In D. Hume (Hrsg.), Political essays (S. 105-114). Cambridge (UK): Cambridge University Press.

Kajetzke, L. (2010). Der Spießer. In S. Moebius \& M. Schroer (Hrsg.), Diven, Hacker, Spekulanten. Sozialfiguren der Gegenwart (S. 366-380). Frankfurt a. M.: Suhrkamp.

Keller, R. (2011). Wissenssoziologische Diskursanalyse. Grundlegung eines Forschungsprogramms. Wiesbaden: VS Verlag für Sozialwissenschaften.

Keller, R., Hirseland, A., Schneider, W., \& Viehöver, W. (Hrsg.). (2001). Handbuch Sozialwissenschaftliche Diskursanalyse. Bd. I: Theorien und Methoden. Wiesbaden: VS Verlag für Sozialwissenschaften.

Klöppel, U. (2010). Foucaults Konzept der Problematisierungsweise und die Analyse diskursiver Transformationen. In A. Landwehr (Hrsg.), Diskursiver Wandel (S. 255-263). Wiesbaden: VS Verlag für Sozialwissenschaften.

Kocka, J. (2001). Das lange 19. Jahrhundert. Arbeit, Nation und bürgerliche Gesellschaft. Stuttgart: KlettCotta.

Kreuzer, H. (2000) [1968]. Die Bohème. Analyse und Dokumentation der intellektuellen Subkultur vom 19. Jahrhundert bis zur Gegenwart. Stuttgart: J. B. Metzler.

Kurbjuweit, D. (2010). Der Wutbürger. Stuttgart 21 und Sarrazin-Debatte: Warum die Deutschen so viel protestieren. Der Spiegel vom 11. Okt. 2010. URL: https:/www.spiegel.de/spiegel/a-724587.html. Zugegriffen: April 2021.

Landwehr, A. (2008). Historische Diskursanalyse. Frankfurt a. M.: Campus.

Magerski, C. (2015). Gelebte Ambivalenz. Die Bohème als Prototyp der Moderne. Wiesbaden: Springer VS.

Mannheim, K. (1982). Ideologische und soziologische Interpretation der geistigen Gebilde. In V. Meja \& N. Stehr (Hrsg.), Der Streit um die Wissenssoziologie. Bd. 1 (S. 213-230). Frankfurt a. M.: Suhrkamp. 
Marg, S. (2014). Mitte in Deutschland. Zur Vermessung eines politischen Ortes. Bielefeld: Transcript.

Marx, K. (1960) [1852]. Der achtzehnte Brumaire des Louis Bonaparte. In K. Marx \& F. Engels, Werke $(M E W)$. Bd. 8 (S. 111-207). Berlin: Dietz.

Marx, K., \& Engels, F. (1959) [1848]. Manifest der Kommunistischen Partei. In K. Marx \& F. Engels, Werke (MEW). Bd. 4 (S. 465-493). Berlin: Dietz.

Meyer, H. (1963). Der Bildungsphilister. In H. Meyer, Zarte Empirie. Studien zur Literaturgeschichte (S. 179-201). Stuttgart: J. B. Metzler.

Misik, R. (2006). Die neuen Spießer. Von der fatalen Sehnsucht nach einer überholten Gesellschaft. Berlin: Ullstein.

Mühsam, E. (1906). Bohême. Die Fackel, 8(202), 4-10.

Mühsam, E. (1978) [1903]. Die Boheme. In E. Mühsam, Briefe an Zeitgenossen. Bd. 2 (S. 7-12). Berlin: Guhl.

Nietzsche, F. (1999) [1873]. Unzeitgemäße Betrachtungen. Erstes Stück: David Strauss, der Bekenner und der Schriftsteller. In F. Nietzsche, Kritische Studienausgabe. Bd. 1 (S. 157-242). München: DTV.

Novalis (1798). Blüthenstaub. Athenaeum, 1(1), 70-106.

Oesterle, G. (1992). Juden, Philister und romantische Intellektuelle. Überlegungen zum Antisemitismus in der Romantik. Athenäum. Jahrbuch der Friedrich-Schlegel-Gesellschaft, 2, 55-89.

Oesterle, G. (Hrsg.). (1997). Jugend, ein romantisches Konzept? Würzburg: Königshausen \& Neumann.

Reckwitz, A. (2006). Das hybride Subjekt. Eine Theorie der Subjektkulturen von der bürgerlichen Moderne zur Postmoderne. Weilerswist: Velbrück.

Schilling, H. (2003). Kleinbürger. Mentalität und Lebensstil. Frankfurt a. M.: Campus.

Schrage, D. (2012). Subjektivierung durch Normalisierung. Zur Aktualisierung eines poststrukturalistischen Konzepts. In B. Riegraf, D. Spreen, \& S. Mehlmann (Hrsg.), Medien - Körper - Geschlecht (S. 73-88). Bielefeld: Transcript.

Schrage, D. (2020). Spottobjekt und Theorieproblem. Marx' Invektiven gegen die Kleinbürger. In E. Mengaldo \& M. Bies (Hrsg.), Marx konkret. Poetik und Ästhetik des Kapitals (S. 23-42). Göttingen: Wallstein.

Schrage, D. (2021). Invektivität und soziale Ordnung. Anschlüsse an Goffmans Konzept der Interaktionsordnung. In A.-L. Garcia, T. Schlinzig, \& R. Simon (Hrsg.), Von Miniaturen bis Gro/sstrukturen. Mikrosoziologie sozialer Ordnung (S. 172-184). Weinheim, Basel: Juventa.

Schulz, A. (2014). Lebenswelt und Kultur des Bürgertums im 19. und 20. Jahrhundert. Berlin, München: De Gruyter Oldenbourg.

Siegrist, H. (1999). Bürgerlichkeit und Antibürgerlichkeit in historischer Perspektive. In G. Meuter \& H. R. Otten (Hrsg.), Der Aufstand gegen den Bürger. Antibürgerliches Denken im 20. Jahrhundert (S. 35-50). Würzburg: Königshausen \& Neumann.

Stein, G. (1985). Philister - Kleinbürger - Spießer. Normalität und Selbstbehauptung. Frankfurt a. M.: Fischer.

Stirner, M. (2016). Der Einzige und sein Eigentum. Freiburg, München: Karl Alber.

Sußebach, H. (2007). Bionade-Biedermeier. Die Zeit vom 7. Nov. 2007. URL: https://www.zeit.de/2007/ 46/D18-PrenzlauerBerg-46. Zugegriffen: April 2021.

Sonja Engel geb. 1980. Dr. des., Wissenschaftliche Mitarbeiterin im Teilprojekt I im Sonderforschungsbereich 1285 „Invektivität. Konstellationen und Dynamiken der Herabsetzung“ an der TU Dresden. Forschungsschwerpunkte: Historische Kultursoziologie, Geschlechtersoziologie. Ausgewählte Veröffentlichungen: (mit D. Schrage) Das Spießerverdikt. Invektiven gegen die Mittelmäßigkeit der Mitte im 19. Jahrhundert, 2021; Georg Simmel: Frühe Abhandlungen zur Theorie der Kultur (bis 1908), in: J. Bohr (Hrsg.), Georg-Simmel-Handbuch, 2021; Kultursoziologie und Geschlecht, in: S. Moebius (Hrsg.), Handbuch Kultursoziologie, 2019.

Dominik Schrage geb. 1969. Dr., Professor für soziologische Theorien und Kultursoziologie an der TU Dresden. Forschungsschwerpunkte: Kultursoziologie, Soziologische Theorien, Historische Soziologie, Soziologie des Konsums und der Medien. Ausgewählte Veröffentlichungen: (mit S. Engel) Das Spießerverdikt. Invektiven gegen die Mittelmäßigkeit der Mitte im 19. Jahrhundert, 2021; (Hrsg. mit H. Schwetter und A.-K. Hoklas), „Zeiten des Aufbruchs“. Populäre Musik als Medium gesellschaftlichen Wandels, 2019; Die Verfügbarkeit der Dinge. Eine historische Soziologie des Konsums, 2009. 